COMMUNICATIONS IN

ANALYSIS AND GEOMETRY

Volume 6, Number 1, 21-65, 1998

\title{
A sharp characterization of the smooth 4-sphere in curvature terms
}

\section{Christophe MARgerin ${ }^{1}$}

This article presents a sharp pointwise geometric characterisation of the smooth structure of $S^{4}$ and its only standard quotient $\mathbb{R} \mathbb{P}^{4}$, which we obtained some years ago. The proof that we give here is a significantly simplified version of our original proof ([M2]). The main statements are the following two theorems.

Theorem 1. Any compact 4-manifold which admits a metric whose curvature is more pinched (w.r.t. "weak pinching", see below) than the product $S^{1} \times\left(S^{3}\right.$, can) is diffeomorphic to the (standard) 4-sphere, if it is orientable, or else to the standard $\mathbb{Z}_{2}$-quotient $\mathbb{R} \mathbb{P}^{4}$.

Its turns out that the products $S^{1} \times\left(S^{3}, \lambda\right.$ can $), \lambda$ in $\mathbb{R}_{+}^{*}$, and the standard projective plane $\left(\mathbb{P}^{2}, \mathrm{~F}-\mathrm{S}\right)$ have the same weak pinching, $1 / 6$. We also prove the rigidity statement that these are basically the only possible limiting examples :

Theorem 2. Any compact 4-manifold which admits a metric whose curvature is as pinched as $S^{1} \times\left(S^{3}\right.$, can) is, if not diffeomorphic to $S^{4}$ or the standard $\mathbb{R P}^{4}$, isometric to either $\left(\mathbb{P}^{2}, F-S\right)$ or a (Riemannian) quotient of $\mathbb{R} \times\left(S^{3}, \lambda\right.$ can $), \lambda \in \mathbb{R}_{+}^{*}$.

At the same time R. Hamilton gave an independant characterization of the smooth $S^{4}$ in terms of positivity of the curvature operator (see [H2]). A key issue lies in the choice of relevant (Riemannian) invariants to work with ; the weak pinching is by definition the norm of the tracefree curvature operator, normalized by the scalar curvature to get a scale invariant,(see Part I for a more formal definition). This concept has been introduced in [M1], where it is also proved that the weak pinching decreases monotonically to zero along any integral curve of the Ricci field ( -2 ric, to be precise), provided that the issuing metric is pinched enough. Note that there the

\footnotetext{
${ }^{1}$ URA 169 du CNRS
} 
assumption is weak enough to allow for the possibility of some negative curvature, and that, on a 4-manifold, 1/6-weak pinching does not imply that the curvature operator is positive, nor vice-versa.

More recently we succeeded in proving a sharp pointwise geometric characterization of the standard differentiable structure of the sphere (and its quotients) in all but a finite number of dimensions. This had been a longstanding conjecture : using different technics, E. Calabi, D. Gromoll, H. Karcher, K. Shiohama, M. Sugimoto and E. Ruh proved some $C^{\circ}{ }^{\circ}$-sphere theorems in the classical setting invoking the ratio of sectional curvatures, but these were still far from the conjectured " $1 / 4$-theorem". This gives us further motivation to publish this new version of our original proof in dimension 4 which, since 1986, has only been available as a preprint (in french). Altogether it should definitely offer weak pinching the geometric status it deserves.

Since of different nature, the higher dimensional cases will not; be discussed in this paper.

It can be noted that in connection with the question of existence of metrics with positive curvature on exotic spheres, Theorems 1 and 2 establish that any metric on the "fake" real projective space constructed by S.E. Cappell and J.L. Shaneson (see [C-S1], [C-S2] and [L]), though a $\mathbb{Z}_{2}$-quotient of the standard 4-sphere, is more distorted than the standard product metric on $S^{1} \times S^{3}$ and the Fubini - Study metric on $\mathbb{P}^{2}$. Moreover, if these is any exotic $S^{4}$ - which is not ruled out by Freedman's solution of the topological 4-dimensional Poincaré conjecture (see $[F]$ ) - this conclusion would also hold for any metric on any such manifold.

The very starting point is Hamilton's result [H1] that any cornpact 3manifold of positive Ricci curvature can be deformed to a manifold of constant sectional curvature. Hamilton's strategy consists in integrating the Ricci curvature as a vector field on the space of metrics of the underlying manifold to get a metric of constant sectional curvature. As early as ' 84 (see [M1]) we demonstrated that this approach could be (somewhat surprisingly) extended to higher dimensions to prove that any sufficiently pinched manifold carries a metric of constant (positive) curvature.

The paper's main contribution is to extend this argument to any metric which is more pinched than the product $S^{1} \times\left(S^{3}\right.$, can $)$, or equivalently, than the standard metric structure on the complex projective plane, ( $\left.\mathbb{P}^{2}, \mathrm{~F}-\mathrm{S}\right)$. By this we mean a metric of positive scalar curvature whose weak pinching is less than the weak pinching of both of these standard structures, i.e. 1/6. It can be noted, in contrast, that the argument can not apply to any of the limit metrics: vanishing of the weak pinching is known to imply constant 
sectional curvature by the second Bianchi identity, which is possible only on (a quotient of) the smooth 4-sphere!

We work only with metrics of positive scalar curvature, (see Paragraph I-C), but it may be worth noticing, as a final comment, that the Ricci curvature of some of the limit metrics, namely of the products $S^{1} \times\left(S^{3}\right.$, can), is not even positive.

Table of contents

I. Notation and prerequisites.

II. Reduction to some problem in real algebraic geometry.
A. Splitting of the fundamental polynomial.
B. Decoupling Ricci and Weyl curvatures.
C. From $\beta$-weak pinching to weak pinching.

III. Solving the algebraic reduction (first steps): a sharp bound for the fundamental polynomial whose dependence on the Ricci curvature is scalar.
A. Some symmetries of the fundamental polynomial.
B. Dependence on the distorsion in the lower two $\widehat{Z}^{2}$-eigenvalues.
C. Dependence on the largest $\widehat{Z}^{2}$ eigenvalue.

IV. Solving the algebraic reduction (continued) : isolating $S^{1} \times S^{3}$-type curvatures.
A. Eliminating some dependence on the Weyl spectrum.
B. Some further elimination w.r.t. the Weyl spectrum and Ricci curvature.

V. Solving the algebraic reduction (end) : isolating $\mathbb{P}^{2}$-type curvatures.
A. Eliminating some dependence on the Weyl spectrum.
B. Eliminating some more dependence : the case where the trace- free Ricci curvature is large.
C. Eliminating some more dependence : the case where the trace- free Ricci curvature is small.

VI. End of the proof.

A. Behavior of the gradient of the scalar curvature. 
B. Geometric conclusion.

VII. Rigidity : classification of the limiting cases.
A. 1/6-weakly pinched manifolds not diffeomorphic to $S^{4}$.
B. Metrics whose curvature is of $S^{1} \times S^{3}$-type.
C. Metrics whose curvature is of $\mathbb{P}^{2}$ or $\overline{\mathbb{P}}^{2}$-type.

\section{Definitions, notations, prerequisites and a first sketch.}

Let $M$ be a compact smooth 4-manifold and $g$ a Riemannian metric on $M$. Using the metric we will freely identify the tangent and cotangent bundles, but for two exceptions : using the metric, we can identify $S^{2}\left(\Lambda^{2} T^{*} M\right)$ with the symmetric endomorphisms of $\Lambda^{2}\left(T^{*} M\right)$; we will denote by ${ }^{-}$the isomorphism. There is also a relevant morphism from $S^{2}\left(\Lambda^{2} T^{*} M\right)$ to the symmetric endomorphisms of $S^{2}\left(T^{*} M\right)$ : let us define for any $(0,4)$-tensor $A$ and vectors $X$ and $Y$,

$$
\stackrel{\circ}{A}(X \otimes Y+Y \otimes X)=A(X, ., Y, .)+A(Y, ., X, .)
$$

and then extend the definition by linearity. We will denote by $(.,$.$) , and |$. the scalar product and the norm on $\otimes^{\bullet} T^{*} M \otimes \otimes^{\bullet} T M$ induced by the metric. Working with endomorphisms, we will denote the identity by id, the trace by $\operatorname{tr}$, the kernel by ker, the set of all eigenvalues by spec and by ${ }^{*}$ the adjunction w.r.t. the scalar product $(.,$.$) .$

$D$ will stand for the unique metric, torsion-free connection on $T M$ as well as for its functorial extension to the tensor algebra $\otimes^{\bullet} T^{*} M$ and $D^{*}$ for its formal $L^{2}$-adjoint on 1-forms, i.e. $D^{*} \alpha=-\sum_{X_{i}}\left(D_{X_{i}} \alpha\right)\left(X_{i}\right)$ for any $g$-orthonormal frame $\left(X_{i}\right)_{i=1}^{\operatorname{dim} M}$.

The Riemannian curvature is then denoted by $R$ (as a tensor field of type $(0,4))$ or by $\widehat{R}$ when we insist on $R$ being a section of $S^{2}\left(\Lambda^{2} T^{*} M\right)$, the bundle of symmetric endomorphisms of the space of skew 2-covectors, or else by $\stackrel{\circ}{R}$, when we view the curvature as a section of $S^{2}\left(S^{2} T^{*} M\right)$, the bundle of symmetric endomorphisms of the space of symmetric 2-vectors. The Ricci trace we denote by ric and the total trace, the scalar curvature, by scal. In the following lines $z$ stands for the tracefree Ricci curvature, $z=\operatorname{ric}-\frac{\text { scal }}{n} g$, and $W$ is the conformal (or Wey l) curvature. We will 
also use a symmetrizing projector onto $S^{2}\left(\Lambda^{2}\left(T^{*} M\right)\right), K$, which we define by the identity:

$$
K(T)=T-\sigma_{2134} \circ T-\sigma_{1243} \circ T+\sigma_{3412} \circ T,
$$

and will denote by $\widehat{K}(T)(\stackrel{\circ}{K}(T))$ when we want to view the result through the isomorphism - (and ${ }^{\circ}$, respectively).

The usual irreducible splitting of the curvature algebra under the $O(n)$ action is written $R=\frac{\text { scal }}{2 n(n-1)} g \circledast g+\frac{z \circledast g}{n-2}+W$ where $\circledast$ is the algebraic lifting from $S^{2} T^{*} M$ to $S^{2} \Lambda^{2} T^{*} M$, sometimes called Kulkarni product although one of the algebraic operations well-known to, and used by invariant theorists in the late nineteen century: for two 2-covectors $h$ and $k$, and four vectors $X_{1}, \ldots, X_{4}$, we set :

$$
\begin{aligned}
h \circledast k\left(X_{1}, X_{2}, X_{3}, X_{4}\right)= & h\left(X_{1}, X_{3}\right) k\left(X_{2}, X_{4}\right)+h\left(X_{2}, X_{4}\right) k\left(X_{1}, X_{3}\right) \\
& -h\left(X_{1}, X_{4}\right) k\left(X_{2}, X_{3}\right)-h\left(X_{2}, X_{3}\right) k\left(X_{1}, X_{4}\right) .
\end{aligned}
$$

We can of course apply previous isomorphisms - and $^{\circ}$ to (the result of) any such product ; we will then denote the composition by $h \widehat{\circledast} k$ and $h \circledast k$ respectively.

The trace-free curvature operator $\mathcal{D}=\frac{z \circledast g}{n-2}+W$ is called the deviation, and its (normalized) pointwise norm $|\mathcal{D}|^{2} \mathrm{scal}^{-2}$, which is invariant under rescaling, weak pinching.

Note that in dimension 4 , one has a further splitting of the Weyl curvature , $W=W^{+}+W^{-}$, when considering the $S O(4)$-action on the curvature algebra which is associated to the Hodge star operator eigendecomposition and reflects the well-known isomorphism $\mathfrak{s o}(4)=\mathfrak{s o}(3) \oplus \mathfrak{s o}(3)$.

Let us recall the following fundamental analytical result upon which our proof relies.

Proposition 1. (Global integrability for the Ricci flow [H1], [M3 chap 2 th. V]) Let $M$ be a compact smooth $n$-manifold and $g_{0}$ any metric on $M$. For all real numbers $\alpha>-\frac{1}{2(n-1)}$, there is a unique maximal integral curve, $g_{t}, t \in[0, T)$, of the vector field -2 (ric $+\alpha$ scal g), originating from $g_{0}$; the $\mathcal{C}^{0}$-norm of the curvature, $R\left(g_{t}\right)$, diverges at $T$ if $T$ is finite.

A proof for the case $\alpha=0$ may be found in [H1]. Since this is enough for our present purposes we will not provide any argument here -although we recommend the more general and natural approach taken in [M3]. 
In the proof of the main theorems we will also need the evolution equations for the basic Riemannian invariants and for weak pinching. The notations are consistent with [M1] where the equations have been first derived.

Proposition 2. (Evolution equation for the curvatures) [M1, Lemma 1 and Proposition 2] Along any integral curve for the field --2 ric the curvatures obey the following differential equations

$$
\begin{aligned}
&\left(\frac{\partial}{\partial t}+D^{*} D\right) \widehat{R}=-\frac{1}{2}(\text { ric } \widehat{\circledast} g-\widehat{R}) \circ \widehat{R} \\
&-\frac{1}{2} \widehat{R} \circ(\text { ric } \widehat{\circledast} g-\widehat{R})+\widehat{K}(\stackrel{\circ}{R} \circ \stackrel{\circ}{R}) ; \\
&\left(\frac{\partial}{\partial t}+D^{*} D\right) \text { ric }=-2 \text { ric } \circ \text { ric }+2 \stackrel{\circ}{R}(\text { ric }) ;
\end{aligned}
$$

and

$$
\left(\frac{\partial}{\partial t}+D^{*} D\right) \text { scal }=2|\mathrm{ric}|^{2} .
$$

Corollary 3. (Evolution equation for weak pinching) [M1, Proposition 2] Along any integral curve for the field -2 ric the weak pinching obeys the following differential equation

(4)

$$
\begin{aligned}
\left(\frac{\partial}{\partial t}+D^{*} D\right) & \left(|\mathcal{D}|^{2} \mathrm{scal}^{-\beta}\right)-2(\beta-1) \mathrm{scal}^{-1}\left(d|\mathcal{D}|^{2} \mathrm{scal}^{-\beta}, d \mathrm{scal}\right)= \\
& -2 \mathrm{scal}^{-2-\beta}|\mathrm{scal} D \mathcal{D}-\mathcal{D} \otimes d \mathrm{scal}|^{2} \\
& -2(2-\beta)(\beta-1) \mathrm{scal}^{-2-\beta}|\mathcal{D}|^{2}|d \mathrm{scal}|^{2} \\
& +2 \mathrm{scal}^{-\beta}\left((\widehat{R} \circ \widehat{R}+\widehat{K}(\stackrel{\circ}{R} \circ \stackrel{\circ}{R}), \widehat{\mathcal{D}})-\beta|\mathcal{D}|^{2}|\mathrm{ric}|^{2} \mathrm{scal}^{-1}\right) .
\end{aligned}
$$

The main body of this paper is devoted to the proof that for any metric (of positive scalar curvature) whose weak pinching is less than $1 / 6$ there is some real number $\bar{\beta}$ in the interval $(1,2)$ s.t. the curvature polynomial

$$
P_{\beta}(R)=(\widehat{R} \circ \widehat{R}+\widehat{K}(\stackrel{\circ}{R} \circ \stackrel{\circ}{R}), \widehat{\mathcal{D}})-\beta|\mathcal{D}|^{2}|\mathrm{ric}|^{2} \mathrm{scal}^{-1}
$$

is non-positive for any real number $\beta$ in the interval $[\bar{\beta}, 2]$; more precisely 
Proposition 4. Let $M$ be a compact smooth 4-manifold. For any metric of positive scalar curvature, $g_{0}$, on $M$ whose weak pinching is less than $1 / 6$ i.e. for any metric which is more pinched than the product $S^{1} \times\left(S^{n-1}\right.$, can $)$ - there is some real number $\beta_{0}$ in the interval $(1,2)$ s.t. for all $\beta$ in $\left[\beta_{0}, 2\right]$ the $\beta$ weak pinching $|\mathcal{D}|^{2} \mathrm{scal}^{-\beta}$ is uniformly bounded along the integral curve of the Ricci field -2 ric starting from $g_{0}$.

In fact, we prove that the global $\beta$-weak pinching $\sup _{V}|\mathcal{D}|^{2} \mathrm{scal}^{-\beta}$ is monotonic along the integral curve, and so $\sup _{V, g_{0}}|\mathcal{D}|^{2}$ scal $^{-\beta^{-}}$is a uniform upper bound along the integral curve of -2 ric originating from $g_{0}$. By the parabolic maximum principle the monotonicity of $\sup _{V, g_{0}}|\mathcal{D}|^{2} \mathrm{scal}^{-\beta}$ reduces to the following statement: the term of differential order 0 in (4), i.e. the fundamental polynomial $P_{2}(R)$, is non-positive on 1/6-weakly pinched curvature tensors, and negative on any more pinched curvature. The latter is largely an algebraic problem.

The solution, i.e. a proof of Proposition 4, spreads over Parts II to V, and must be considered the heart of the paper. The strategy to derive the geometric conclusions from Proposition 4 is not new ; for completeness's sake, we give the details in Part VI, which consists basically in a specialization in dimension 4 of arguments in [M1, Proposition 3]. Part VII is devoted to the rigidity theorem (Theorem 2) that characterizes the limit geometries. This part does rely on Theorem 1 and its proof, since exact 1/6-weak pinching is too weak an assumption to characterize the limit geometries: there are (exactly) 1/6-weakly pinched geometries on $S^{4}$, for example, obtained from the canonical metric by appropriate bashes. Using technics involved in proving Theorem 1 we can nevertheless prove that from the curvature viewpoint there are basically only two types of $1 / 6$-weakly pinched geometries which are not diffeomorphic to $S^{4}$ nor $\mathbb{R} \mathbb{P}^{4}$. In both cases, the curvatures offer enough symmetries to enable us to reconstruct the geometry from the curvature "à la Elie Cartan". Although different from the discussion of the limit cases in [H2], there are expected similarities when reconstructing the limit geometries from the holonomy reductions, like in Lemma 36 below. Note once more 1/6-weak pinching does not imply non-negativity for the curvature operator, which makes life harder at this stage also, and that we can reconstruct the projective plane without invoking the Frankel conjecture. 
II. Reduction to a problem in real algebraic geometry.

\section{A. A splitting of the fundamental polynomial.}

We begin with the additional splitting of the fundamental polynomial $P_{\beta}(R)$ associated to the $\mathfrak{s o}(4)$-splitting of the Weyl algebra according to the eigenspaces of the Hodge $*$-operator. The Riemannian curvature tensor of a 4-manifold, when viewed as a (symmetric) endomorphism of $\Lambda^{2} T^{*} M$ splits into a (symmetric) endomorphism of $\Lambda^{+}, \widehat{R}^{+}=\frac{\text { scal }}{6} \mathrm{id}_{\Lambda^{+}}+\widehat{W}^{+}$, where $\widehat{W}^{+}$is the restriction $\widehat{W}_{\mid \Lambda^{+}}$, a (symmetric) endomorphism of $\Lambda^{--}, \widehat{R}^{-}=$ $\frac{\text { scal }}{6} \mathrm{id}_{\Lambda^{-}}+\widehat{W}^{-}, \widehat{W}^{-}=\widehat{W}_{\mid \Lambda^{-}}$a morphism from $\Lambda^{+}$to $\Lambda^{-}, \widehat{Z}_{\mid \Lambda^{+}}=\left.\frac{z \widehat{\oplus} g}{2}\right|_{\Lambda^{+}}$, and its transpose (w.r.t. the natural scalar product on $\Lambda^{2} T^{*} M$ ) from $\Lambda^{-}$ to $\Lambda^{+},\left.\frac{z \hat{\oplus} g}{2}\right|_{\Lambda^{-}}$. Introducing the (skew-)commutator of two endomorphisms, $[.,$.$] and \{.,$.$\} respectively, we can summarize this in the following identities$

$$
\widehat{R}=\frac{\text { scal }}{6} \mathrm{id}+\widehat{Z}+\widehat{W}, \quad\{\widehat{Z}, *\}=0
$$

and

$$
[\widehat{W}, *]=0
$$

For convenience, set $\widehat{Z}_{\mid \Lambda^{-}}=B$ and for any linear morphism $u$ between two linear spaces of the same dimension $n$, denote by $\tilde{u}$ the cotranspose of $u$ i.e. the unique linear map defined by

$$
\tilde{u}(x) \wedge y_{2} \wedge \cdots \wedge y_{n}=x \wedge u\left(y_{2}\right) \wedge \cdots \wedge u\left(y_{n}\right)
$$

To avoid clumsy notations, we also denote by the composition of with the - isomorphism.

According to the previous splitting, the fundamental polynomial $P_{\beta}(R)$ can be rewritten 


\section{Lemma 5.}

(6)

$$
\begin{aligned}
P_{\beta}(R)= & \operatorname{tr}\left(\widehat{W}^{+}\right)^{3}+\operatorname{tr}\left(\widehat{W}^{-}\right)^{3}+3\left(B B^{*}, \widehat{W}^{+}\right)+3\left(B B^{*}, \widehat{W}^{-}\right) \\
& +\frac{\text { scal }}{3}\left(\operatorname{tr}_{\Lambda^{+}} B B^{*}+\operatorname{tr}_{\Lambda^{-}} B^{*} B\right) \\
& +\frac{\text { scal }}{3}\left(\operatorname{tr}\left(\widehat{W}^{+}\right)^{2}+\operatorname{tr}\left(\widehat{W}^{-}\right)^{2}\right) \\
& +2\left(\widetilde{R}^{+}, \widehat{R}^{+}-\frac{\mathrm{scal}}{6} \mathrm{id}_{\Lambda^{+}}\right)+2\left(\widetilde{R}^{-}, \widehat{R}^{-}-\frac{\mathrm{scal}}{6} \mathrm{id}_{\Lambda^{-}}\right) \\
& -2\left(\widetilde{B}, B^{*}\right)-2\left(\widetilde{B}^{*}, B\right)-2|\mathcal{D}|^{2}|\mathrm{ric}|^{2} \mathrm{scal}^{-1} \\
& +(2-\beta)|\mathcal{D}|^{2}|\mathrm{ric}|^{2} \mathrm{scal}^{-1} .
\end{aligned}
$$

Proof. Let $\left(e_{i}\right)_{i=1}^{n}$ be an orthonormal moving frame (for $T M$ ), so that $\varepsilon_{1}^{ \pm}=$ $e_{1} \wedge e_{2} \pm e_{3} \wedge e_{4}, \varepsilon_{2}^{ \pm}=e_{1} \wedge e_{3} \mp e_{2} \wedge e_{4}$ and $\varepsilon_{3}^{ \pm}=e_{1} \wedge e_{4} \pm e_{2} \wedge e_{3}$ form an orthogonal basis for $\Lambda^{+}$(resp. $\Lambda^{-}$). One can then check the following identities

$$
\begin{aligned}
K(\stackrel{\circ}{R} \circ \stackrel{\circ}{R})\left(\varepsilon_{1}^{+}, \varepsilon_{1}^{+}\right) & =2^{-1}\left(\widehat{R}\left(\varepsilon_{2}^{+}\right), \varepsilon_{2}^{+}\right)\left(\widehat{R}\left(\varepsilon_{3}^{+}\right), \varepsilon_{3}^{+}\right)-\left(\widehat{R}\left(\varepsilon_{2}^{+}\right), \varepsilon_{3}^{+}\right)^{2} \\
& =2\left(\widetilde{R}^{+}\left(\varepsilon_{1}^{+}\right), \varepsilon_{1}^{+}\right)
\end{aligned}
$$

By similar calculations, one can derive the following identities

$$
\begin{aligned}
K(\stackrel{\circ}{R} \circ \stackrel{\circ}{R})\left(\varepsilon_{i}^{+}, \varepsilon_{j}^{+}\right) & =2\left(\widetilde{R}^{+}\left(\varepsilon_{i}^{+}\right), \varepsilon_{j}^{+}\right)=2\left(\widetilde{R}^{+}\left(\varepsilon_{j}^{+}\right), \varepsilon_{i}^{+}\right) ; \\
K(\stackrel{\circ}{R} \circ \stackrel{\circ}{R})\left(\varepsilon_{i}^{-}, \varepsilon_{j}^{-}\right) & =2\left(\widetilde{R}^{+}\left(\varepsilon_{i}^{-}\right), \varepsilon_{j}^{-}\right)=2\left(\widetilde{R}^{+}\left(\varepsilon_{j}^{-}\right), \varepsilon_{i}^{-}\right) ; \\
K(\stackrel{\circ}{R} \circ \stackrel{\circ}{R})\left(\varepsilon_{i}^{-}, \varepsilon_{j}^{+}\right) & =K(\stackrel{\circ}{R} \circ \stackrel{\circ}{R})\left(\varepsilon_{j}^{+}, \varepsilon_{i}^{-}\right)=-2\left(\widetilde{B}^{*}\left(\varepsilon_{i}^{-}\right), \varepsilon_{j}^{+}\right) \\
& =-2\left(\widetilde{B}\left(\varepsilon_{j}^{+}\right), \varepsilon_{i}^{-}\right) .
\end{aligned}
$$


This can be written

$$
\begin{aligned}
& \Pi_{\left(\Lambda^{+}\right)^{*} \otimes \Lambda^{+}} \widehat{K}(\stackrel{\circ}{R} \circ \stackrel{\circ}{R})=2 \widetilde{R}^{+} ; \\
& \Pi_{\left(\Lambda^{+}\right)^{*} \otimes \Lambda^{-}} \widehat{K}(\stackrel{\circ}{R} \circ \stackrel{\circ}{R})=-2 \widetilde{B} ; \\
& \Pi_{\left(\Lambda^{-}\right)^{*} \otimes \Lambda^{+}} \widehat{K}(\stackrel{\circ}{R} \circ \stackrel{\circ}{R})=-2 \widetilde{B}^{*} ; \\
& \Pi_{\left(\Lambda^{-}\right)^{*} \otimes \Lambda^{-}} \widehat{K}(\stackrel{\circ}{R} \circ \stackrel{\circ}{R})=2 \widetilde{R}^{-}
\end{aligned}
$$

where $\Pi_{E}$ stands for the orthogonal projection onto $E$, (and $E$ is here a subset of End $\left.\left(\Lambda^{2} \mathrm{~T}^{*} \mathrm{M}\right)\right)$.

\section{B. Decoupling Ricci and Weyl curvatures.}

As symmetric endomorphisms of $\Lambda^{+}$(resp. $\Lambda^{-}$), $\widehat{W}^{+}$and $B B^{*}$ (resp. $\widehat{W}^{-}$and $B^{*} B$ ) are diagonalizable on $\mathbb{R}$ in orthonormal bases. Let $\left(r_{i}^{+}\right)_{i=1}^{3}, r_{1}^{+} \leq r_{2}^{+} \leq r_{3}^{+}$be the spectrum of $\widehat{R}^{+},\left(r_{i}^{-}\right)_{i=1}^{3}, r_{1}^{-} \leq r_{2}^{-} \leq r_{3}^{-}$, the spectrum of $\widehat{R}^{-}$, and $\left(b_{i}^{2}\right)_{i=1}^{3}, 0 \leq b_{1} \leq b_{2} \leq b_{3}$ the $B^{*} B$ (and $B B^{*}$ )spectrum.

Lemma 6. Let $r^{+}=\Sigma_{i=1}^{3} r_{i}^{+}\left(r^{-}=\Sigma_{i=1}^{3} r_{i}^{-}\right)$be the trace of $\hat{R}^{+}$(resp. $\left.\widehat{R}^{-}\right)$. The scalar products $\left(B B^{*}, \widehat{W}^{+}\right)$and $\left(B^{*} B, \widehat{W}^{-}\right)$admit the following sharp upper bounds :

$$
\begin{aligned}
& \left(B B^{*}, \widehat{W}^{+}\right) \leq \sum_{i=1}^{3}\left(r_{i}^{+}-\frac{r^{+}}{3}\right) b_{i}^{2} \\
& \left(B^{*} B, \widehat{W}^{-}\right) \leq \sum_{i=1}^{3}\left(r_{i}^{-}-\frac{r^{-}}{3}\right) b_{i}^{2} .
\end{aligned}
$$

Proof. More generally, let $\varphi$ and $\psi$ be two real endomorphisms and suppose that $\varphi$ is diagonalizable on $\mathbb{R}$ in an orthonormal basis and that the spectrum of $\psi$ is real. Denote by $\lambda_{1} \leq \cdots \leq \lambda_{n}$ the $\varphi$-spectrum and by $\mu_{1} \leq \cdots \leq$ $\mu_{n}$ the $\psi$-spectrum and consider an orthonormal eigenbasis for $\varphi,\left(e_{i}\right)_{i=1}^{n}$, 
associated with the eigenvalues $\left(\lambda_{i}\right)_{i=1}^{n}$. Then

$$
\begin{aligned}
(\varphi, \psi) & =\sum_{i=1}^{n} \lambda_{i}\left(\psi\left(e_{i}\right), e_{i}\right) \\
& =\sum_{i=1}^{n} \lambda_{i} \mu_{i}-\sum_{i=1}^{n-1}\left(\lambda_{i+1}-\lambda_{i}\right)\left(\sum_{j=i+1}^{n} \mu_{j}-\sum_{j=i+1}^{n}\left(\psi\left(e_{j}\right), e_{j}\right)\right) \\
& \leq \sum_{i=1}^{n} \lambda_{i} \mu_{i}
\end{aligned}
$$

Let us define $\Delta_{r^{+}}=\left(r_{3}^{+}-r_{2}^{+}\right)^{2}+\left(r_{2}^{+}-r_{1}^{+}\right)^{2}+\left(r_{3}^{+}-r_{1}^{+}\right)^{2}$ and similarly $\Delta_{r^{-}}$with respect to the $r_{i}^{-}$, and introduce the curvature polynomial

$$
\begin{aligned}
Q_{\beta}(R)= & -\left(\left(r_{3}^{+}-r_{1}^{+}\right)^{2} r_{2}^{+}+\left(r_{3}^{+}-r_{2}^{+}\right)^{2} r_{1}^{+}+\left(r_{2}^{+}-r_{1}^{+}\right)^{2} r_{3}^{+}\right. \\
& \left.+\left(r_{3}^{-}-r_{1}^{-}\right)^{2} r_{2}^{-}+\left(r_{3}^{-}-r_{2}^{-}\right)^{2} r_{1}^{-}+\left(r_{2}^{-}-r_{1}^{-}\right)^{2} r_{3}^{-}\right) \\
& +3 \sum_{i=1}^{3}\left(r_{i}^{+}+r_{i}^{-}\right) b_{i}^{2}-\frac{8}{3} r \sum_{i=1}^{3} b_{i}^{2}-2 r^{-1}\left(\sum_{i=1}^{3} b_{i}^{2}\right)^{2} \\
& +12 b_{1} b_{2} b_{3}-\left(\frac{\Delta_{r^{+}}}{3}+\frac{\Delta_{r^{-}}}{3}\right) r^{-1} \sum_{i=1}^{3} b_{i}^{2} \\
& +(2-\beta)|\mathcal{D}|^{2}\left(|z|^{2}+\frac{\mathrm{scal}^{2}}{4}\right) \mathrm{scal}^{-1} .
\end{aligned}
$$

Corollary 7. The fundamental polynomial $P_{\beta}(R)$ admits $Q_{\beta}(R)$ as a sharp upper bound. It is obtained by decoupling the Weyl and Ricci curvatures in $P_{\beta}(R)$.

Proof. The first Bianchi identity amounts to

$$
r^{+}-r^{-}=12\left(\Pi_{\Lambda^{4}} R\right)=0 ;
$$

Let us denote $r=r^{+}\left(=r^{-}\right)$. The following three identities are then easily derived:

$$
|Z|^{2}=\frac{1}{4}|z \circledast g|^{2}=2|z|^{2}=\operatorname{tr} B B^{*}+\operatorname{tr} B^{*} B=2 \sum_{i=1}^{3} b_{i}^{2} ;
$$


(b)

$$
\Delta_{x}=3 \sum_{i=1}^{3}\left(x_{i}-\frac{x}{3}\right)^{2}, x \in\left\{r^{+}, r^{-}\right\}
$$

(c)

$$
\begin{aligned}
\sum_{i=1}^{3}\left(x_{i}-\frac{x}{3}\right)^{3}+ & 6 x_{1} x_{2} x_{3}-\frac{2 x}{3}\left(x_{1} x_{2}+x_{2} x_{3}+x_{1} x_{3}\right) \\
+ & \frac{2 x}{3} \sum_{i=1}^{3}\left(x_{i}-\frac{x}{3}\right)^{2}-\sum_{i=1}^{3}\left(x_{i}-\frac{x}{3}\right)^{2} x \\
= & -\left(x_{1}\left(x_{3}-x_{2}\right)^{2}+x_{2}\left(x_{3}-x_{1}\right)^{2}\right. \\
& \left.+x_{3}\left(x_{2}-x_{1}\right)^{2}\right), \text { where } x \in\left\{r^{+}, r^{-}\right\}
\end{aligned}
$$

These imply the following upper bound for $P_{\beta}(R)$

(10)

$$
\begin{aligned}
P_{\beta}(R) \leq & -\left(\left(r_{3}^{+}-r_{1}^{+}\right)^{2} r_{2}^{+}+\left(r_{3}^{+}-r_{2}^{+}\right)^{2} r_{1}^{+}+\left(r_{2}^{+}-r_{1}^{+}\right)^{2} r_{3}^{+}\right. \\
& \left.+\left(r_{3}^{-}-r_{1}^{-}\right)^{2} r_{2}^{-}+\left(r_{3}^{-}-r_{2}^{-}\right)^{2} r_{1}^{-}+\left(r_{2}^{-}-r_{1}^{-}\right)^{2} r_{3}^{-}\right) \\
& +3 \sum_{i=1}^{3}\left(r_{i}^{+}+r_{i}^{-}\right) b_{i}^{2}-\frac{8}{3} r \sum_{i=1}^{3} b_{i}^{2}-2 r^{-1}\left(\sum_{i=1}^{3} b_{i}^{2}\right)^{2} \\
& +12 b_{1} b_{2} b_{3}-\left(\frac{\Delta_{r^{+}}}{3}+\frac{\Delta_{r^{-}}}{3}\right) r^{-1} \sum_{i=1}^{3} b_{i}^{2} \\
& +(2-\beta)|\mathcal{D}|^{2}\left(|z|^{2}+\frac{\mathrm{scal}^{2}}{4}\right) \mathrm{scal}^{-1} .
\end{aligned}
$$

\section{Reduction : from $\beta$-weak pinching to weak pinching.}

Because of the differential equation satisfied by the scalar curvature along any integral curve of the Ricci curvature (see (3)), the maximum principle implies that positive scalar curvature is preserved ; in particular, scalar curvature never vanishes along any integral curve originating from. a metric of positive scalar curvature. One may introduce the following homogeneous (scale invariant) curvature-type invariants:

$\breve{r}_{i}^{+}=r_{i}^{+} r^{-1}, \breve{r}_{i}^{-}=r_{i}^{-} r^{-1}, \breve{b}_{i}=b_{i} r^{-1}, \breve{\Delta}_{r^{+}}=r^{-2} \Delta_{r^{+}}$and $\breve{\Delta}_{r^{-}}=r^{-2} \Delta_{r^{-}}$. 
The curvature polynomial $Q_{\beta}$ may then be written with respect to these new variables as

$$
\begin{aligned}
Q_{\beta}(R)= & r^{3}\left(-\left(\breve{r}_{3}^{+}-\breve{r}_{2}^{+}\right)^{2} \breve{r}_{1}^{+}-\left(\breve{r}_{3}^{+}-\breve{r}_{1}^{+}\right)^{2} \breve{r}_{2}^{+}-\left(\breve{r}_{2}^{+}-\breve{r}_{1}^{+}\right)^{2} \breve{r}_{3}^{+}\right. \\
& \left.-\left(\breve{r}_{3}^{-}-\breve{r}_{2}^{-}\right)^{2} \breve{r}_{1}^{-}-\left(\breve{r}_{3}^{-}-\breve{r}_{1}^{-}\right)^{2} \breve{r}_{2}^{-}-\left(\breve{r}_{2}^{-}-\breve{r}_{1}^{-}\right)^{2} \breve{r}_{3}^{-}\right) \\
& +3 \sum_{i=1}^{3}\left(\breve{r}_{i}^{+}+\breve{r}_{i}^{-}\right) \breve{b}_{i}^{2}-\frac{8}{3} \sum_{i=1}^{3} \breve{b}_{i}^{2} \\
& \left.-2\left(\sum \breve{b}_{i}^{2}\right)^{2}+12 \breve{b}_{1} \breve{b}_{2} \breve{b}_{3}-\frac{\left(\breve{\Delta}_{r^{+}}+\breve{\Delta}_{r^{-}}\right)}{3} \sum_{i=1}^{3} \breve{b}_{i}^{2}\right) \\
& +(2-\beta)|\mathcal{D}|^{2} \mathrm{scal}^{-2}\left(|z|^{2} \mathrm{scal}^{-2}+1 / 4\right) \mathrm{scal}^{3}
\end{aligned}
$$

First let us note that, although the curvature polynomial $Q_{\beta}(R)$ does vanish for constant curvature $(\mathcal{D} \equiv 0)$, it is non-positive for very pinched curvature :

Lemma 8. If the weak pinching is small enough, $Q_{\beta}(R)$ is non-positive for all $\beta$ sufficiently close to 2 ; more precisely

$$
\begin{gathered}
\exists \varepsilon_{0}>0 \text { s.t. } \forall \varepsilon, 0<\varepsilon \leq \varepsilon_{0}, \exists \beta_{0}, 0<\beta_{0}<2 \text { s.t. } \\
|\mathcal{D}|^{2} \text { scal }^{-2} \leq \varepsilon^{2} \Rightarrow Q_{\beta}(R) \leq 0, \forall \beta \in\left[\beta_{0}, 2\right] .
\end{gathered}
$$

Proof. It amounts to staightforward arithmetic. Assuming

$$
|\mathcal{D}|^{2} \text { scal }^{-2}\left(=\frac{1}{12}\left(\breve{\Delta}_{r^{+}}+\breve{\Delta}_{r^{-}}\right)+\frac{1}{2}\left(\sum_{i=1}^{3} \breve{b}_{i}^{2}\right)\right) \leq \varepsilon^{2},
$$

we can check the following bounds

(a)

$$
\begin{aligned}
3 \sum_{i=1}^{3}\left(\breve{r}_{i}^{+}+\breve{r}_{i}^{-}\right) \breve{b}_{i}^{2} \leq & 2 \sum_{i=1}^{3} \breve{b}_{i}^{2} \\
& +3\left(\sum_{i=1}^{3}\left(\breve{r}_{i}^{+}-1 / 3\right)^{2}+\Sigma\left(\breve{r}_{i}^{-}-1 / 3\right)^{2}\right)\left(\sum_{i=1}^{3} \breve{b}_{i}^{4}\right)^{1 / 2} \\
\leq & 2(1+3 \sqrt{2} \varepsilon) \sum_{i=1}^{3} \breve{b}_{i}^{2}
\end{aligned}
$$


(b)

$$
\breve{b}_{1} \breve{b}_{2} \breve{b}_{3} \leq\left(\sum_{i=1}^{3} \breve{b}_{i}^{2}\right) \frac{1}{3 \sqrt{3}}
$$

(c)

$$
\begin{aligned}
\left(\breve{r}_{3}^{+}-\breve{r}_{1}^{+}\right)^{2} \breve{r}_{2}^{+}+ & \left(\breve{r}_{3}^{+}-\breve{r}_{2}^{+}\right)^{2} \breve{r}_{1}^{+}+\left(\breve{r}_{2}^{+}-\breve{r}_{1}^{+}\right)^{2} \breve{r}_{3}^{+} \\
= & \frac{1}{3} \breve{\Delta}_{r^{+}}+\left(\breve{r}_{3}^{+}-\breve{r}_{2}^{+}\right)^{2}\left(\breve{r}_{2}^{+}-1 / 3\right) \\
& +\left(\breve{r}_{3}^{+}-\breve{r}_{2}^{+}\right)^{2}\left(\breve{r}_{1}^{+}-1 / 3\right)+\left(\breve{r}_{2}^{+}-\breve{r}_{1}^{+}\right)^{2}\left(\breve{r}_{3}^{+}-1 / 3\right) \\
\geq & \frac{1}{3} \breve{\Delta}_{r^{+}}(1-6 \varepsilon),
\end{aligned}
$$

using Hölder inequality, and similarly,

(c')

$$
\left(\breve{r}_{3}^{-}-\breve{r}_{1}^{-}\right)^{2} \breve{r}_{2}^{-}+\left(\breve{r}_{3}^{-}-\breve{r}_{2}^{-}\right)^{2} \breve{r}_{1}^{-}+\left(\breve{r}_{2}^{-}-\breve{r}_{1}^{-}\right)^{2} \breve{r}_{3}^{-} \geq \frac{1}{3} \breve{\Delta}_{r^{-}}(1-6 \varepsilon) .
$$

From these, we deduce the following upper bound for $Q_{\beta}(R)$ :

$$
\begin{aligned}
Q_{\beta}(R) \leq r^{3}( & -\frac{\breve{\Delta}_{r^{+}}}{3}\left(1-6 \varepsilon-(1-\beta / 2)\left(1+2 \varepsilon^{2}\right)\right) \\
& \left.-\frac{\breve{\Delta}_{r^{-}}}{3}\left(1-6 \varepsilon-(1-\beta / 2)\left(1+2 \varepsilon^{2}\right)\right)\right) \\
& -\left(\sum_{i=1}^{3} \breve{b}_{i}^{2}\right)\left(\frac{2}{3}-6 \sqrt{2} \varepsilon-\frac{4 \sqrt{2}}{\sqrt{3}} \varepsilon-(2-\beta)\left(1+2 \varepsilon^{2}\right)\right)
\end{aligned}
$$

which leads to the lemma.

Because of this, we can isolate the very pinched curvatures and prove by some compactness argument that for any pinching constant $C, C \in(0,1 / 6)$, there is some positive constant $\beta_{0}(C), \beta_{0}(C) \in(1,2)$, such that $Q_{\beta}(R)$ is non-positive for all $\beta \in\left[\beta_{0}, 2\right]$ and $R$ s.t. $Q_{2}(R)<0$ :

Lemma 9. Suppose the curvature polynomial $Q_{2}(R)$ is negative for all $R$ such that $0<|\mathcal{D}|^{2} \mathrm{scal}^{-2}<1 / 6$. Then for any positive real number $\delta$ there is some positive number $\beta_{0}$ s.t. $Q_{\beta}(R)$ is non-positive for every real $\beta$ in the interval $\left[\beta_{0}, 2\right]$ and every curvature-type tensor $R$ s.t. $|\mathcal{D}|^{2}$ scal $^{-2} \leq 1 / 6-\delta$.

Proof. According to Lemma 8, there exist two positive real numbers $\varepsilon$ and $\beta_{1}, \beta_{1}<2$, s.t. $Q_{\beta}(R)$ is non-positive for $\beta$ in $\left[\beta_{1}, 2\right]$ and $|\mathcal{D}|^{2}$ scal $^{-2} \leq \varepsilon$. 
The equations $\sum_{i=1}^{3} \breve{r}_{i}^{+}=1, \sum_{i=1}^{3} \breve{r}_{i}^{-}=1$ and the inequalities $0 \leq \breve{b}_{1} \leq$ $\breve{b}_{2} \leq \breve{b}_{3}, \breve{r}_{1}^{+} \leq \breve{r}_{2}^{+} \leq \breve{r}_{3}^{+}, \breve{r}_{1}^{-} \leq \breve{r}_{2}^{-} \leq \breve{r}_{3}^{-}$and $4 \varepsilon \leq \sum_{i=1}^{3}\left(\breve{r}_{i}^{+}-1 / 3\right)^{2}+$ $\left(\breve{r}_{i}^{-}-1 / 3\right)^{2}+2 \breve{b}_{i}^{2} \leq 4(1 / 6-\delta)$ define a compact semi-algebraic variety, $D_{\varepsilon}^{\delta}$, in $\mathbb{R}^{9}$ on which $r^{-3} Q_{2}$ achieves its negative maximum $m$. Since

$$
\begin{aligned}
Q_{\beta}(R) & =r^{3}\left(r^{-3} Q_{2}(R)+8(2-\beta)|\mathcal{D}|^{2} \mathrm{scal}^{-2}\left(|z|^{2} \mathrm{scal}^{-2}+1 / 4\right)\right) \\
& \leq r^{3}\left(r^{-3} Q_{2}(R)+\frac{4(2-\beta)}{9}\right)
\end{aligned}
$$

$Q_{\beta}(R)$ is non-positive on $D_{\varepsilon}^{\delta}$ for all $\beta$ greater than or equal to $2+\frac{9 m}{4}$. We conclude the proof by setting $\beta_{0}=\max \left(\beta_{1}, 2+\frac{9 m}{4}\right)$.

\section{Solving the algebraic reduction: reduction of the dependence of the fundamental polynomial on the Ricci curvature to a dependence on its norm.}

In the previous paragraph we have reduced the proof of Proposition 4 to the statement that the real semi-algebraic variety of (formal) codimension 2 in $\mathbb{R}^{9}$, defined by the following equations and inequalities is empty :

$$
\left\{\begin{array}{l}
\breve{r}_{1}^{+}+\breve{r}_{2}^{+}+\breve{r}_{3}^{+}=\breve{r}_{1}^{-}+\breve{r}_{2}^{-}+\breve{r}_{3}^{-}=1, \breve{r}_{1}^{+} \leq \breve{r}_{2}^{+} \leq \breve{r}_{3}^{+}, \breve{r}_{1}^{-} \leq \breve{r}_{2}^{-} \leq \breve{r}_{3}^{-} \\
0 \leq \breve{b}_{1} \leq \breve{b}_{2} \leq \breve{b}_{3}, 0 \leq \breve{\Delta}_{r^{+}}+\breve{\Delta}_{r^{-}}+6|\breve{B}|^{2}<2 \text { and } \\
-3\left(\left(\breve{r}_{3}^{+}-\breve{r}_{2}^{+}\right)^{2} \breve{r}_{1}^{+}+\left(\breve{r}_{3}^{+}-\breve{r}_{1}^{+}\right)^{2} \breve{r}_{2}^{+}+\left(\breve{r}_{2}^{+}-\breve{r}_{1}^{+}\right)^{2} \breve{r}_{3}^{+}\right. \\
\left.+\left(\breve{r}_{3}^{-}-\breve{r}_{2}^{-}\right)^{2} \breve{r}_{1}^{-}+\left(\breve{r}_{3}^{-}-\breve{r}_{1}^{-}\right)^{2} \breve{r}_{2}^{-}+\left(\breve{r}_{2}^{-}-\breve{r}_{1}^{-}\right)^{2} \breve{r}_{3}^{-}\right) \\
+9\left(\left(\breve{r}_{3}^{+}+\breve{r}_{3}^{-}\right) \breve{b}_{3}^{2}+\left(\breve{r}_{2}^{+}+\breve{r}_{2}^{-}\right) \breve{b}_{2}^{2}+\left(\breve{r}_{1}^{+}+\breve{r}_{1}^{-}\right) \breve{b}_{1}^{2}\right) \\
-8|\breve{B}|^{2}-6|\breve{B}|^{4}+36 \breve{b}_{1} \breve{b}_{2} \breve{b}_{3}-|\breve{B}|^{2}\left(\breve{\Delta}_{r^{+}}+\breve{\Delta}_{r^{-}}\right) \geq 0 .
\end{array}\right.
$$

Let us recall that the auxiliary variables $\breve{b}_{i}$, and $\breve{\Delta}_{x}=\left(\breve{x}_{3}-\breve{x}_{2}\right)^{2}+$ $\left(\breve{x}_{3}-\breve{x}_{1}\right)^{2}+\left(\breve{x}_{2}-\breve{x}_{1}\right)^{2}, x \in r^{+}, r^{-}$have been introduced earlier, and that consistently we set here $\breve{B}=r^{-1} B$ so that the identity $|\breve{B}|^{2}=\sum_{i=1}^{3} \breve{b}_{i}^{2}$ holds.

No classical algebraic inequality nor any rough variational approach with respect to any natural geometric parameter can solve the reduction. This is due to the existence of two different curvature tensors, of type $S^{1} \times S^{3}$ and $\mathbb{P}^{2}$ respectively, that both lie on the boundary of the previous variety. Because of this bifurcation, variational and/or convexity arguments are only 
possible on some part of the variety under consideration. Moreover these convexity arguments often require some auxiliary variables which reflect in a better way the symmetries of the algebraic system: the geometrical content of the variables may help to discover them. This is made more explicit in the following three parts.

\section{A. Some symmetries of the fundamental polynomial.}

Let us introduce the auxiliary variables $\bar{r}_{i}^{+}, \bar{r}_{i}^{-}$, defined by the identities

$$
\breve{r}_{i}^{+}=\bar{r}_{i}^{+}+\frac{1}{3}, \quad \breve{r}_{i}^{-}=\bar{r}_{i}^{-}+\frac{1}{3},
$$

$\alpha_{1}, \alpha_{2}$, defined by the identities

$$
\bar{r}_{1}^{+}=\frac{1}{\sqrt{6}} \alpha_{1}+\frac{1}{\sqrt{2}} \alpha_{2}, \quad \bar{r}_{2}^{+}=\frac{1}{\sqrt{6}} \alpha_{1}-\frac{1}{\sqrt{2}} \alpha_{2},
$$

and $\gamma_{1}$ and $\gamma_{2}$ which are defined similarly with respect to $\bar{r}_{1}^{-}$and $\bar{r}_{2}^{-}$.

Lemma 10. The following identities hold :

$\bar{r}_{3}^{+}=-\frac{2}{\sqrt{6}} \alpha_{1}, \bar{r}_{3}^{-}=-\frac{2}{\sqrt{6}} \gamma_{1}, \breve{\Delta}_{r^{+}}=3\left(\alpha_{1}^{2}+\alpha_{2}^{2}\right)$ and $\breve{\Delta}_{r^{-}}=3\left(\gamma_{1 .}^{2}+\gamma_{2}^{2}\right)$.

The inequalities $\bar{r}_{1}^{+} \leq \bar{r}_{2}^{+} \leq \bar{r}_{3}^{+}$and $\bar{r}_{1}^{-} \leq \bar{r}_{2}^{-} \leq \bar{r}_{3}^{-}$are equivalent to $\sqrt{3} \alpha_{1} \leq$ $\alpha_{2} \leq 0$ and $\sqrt{3} \gamma_{1} \leq \gamma_{2} \leq 0$, respectively. Finally the curvature polynomial

$$
\left(\breve{r}_{3}^{+}-\breve{r}_{2}^{+}\right)^{2} \breve{r}_{1}^{+}+\left(\breve{r}_{3}^{+}-\breve{r}_{1}^{+}\right)^{2} \breve{r}_{2}^{+}+\left(\breve{r}_{2}^{+}-\breve{r}_{1}^{+}\right)^{2} \breve{r}_{3}^{+}
$$

becomes

$$
\frac{1}{3} \breve{\Delta}_{r^{+}}+\frac{3}{\sqrt{6}} \alpha_{1}^{3}-\frac{9}{\sqrt{6}} \alpha_{1} \alpha_{2}^{2}
$$

and similarly,

$$
\left(\breve{r}_{3}^{-}-\breve{r}_{2}^{-}\right)^{2} \breve{r}_{1}^{-}+\left(\breve{r}_{3}^{-}-\breve{r}_{1}^{-}\right)^{2} \breve{r}_{2}^{-}+\left(\breve{r}_{2}^{-}-\breve{r}_{1}^{-}\right)^{2} \breve{r}_{3}^{-}=\frac{1}{3} \breve{\Delta}_{r^{-}}+\frac{3}{\sqrt{6}} \gamma_{1}^{3}-\frac{9}{\sqrt{6}} \gamma_{1} \gamma_{2}^{2}
$$

Proof. This can be checked by straightforward substitutions and simplifications. 
Corollary 11. The algebraic reduction (11) is equivalent to the polynomial

$$
\begin{aligned}
S_{1}(R)= & -3\left(\alpha_{1}^{2}+\alpha_{2}^{2}+\gamma_{1}^{2}+\gamma_{2}^{2}\right)-\frac{9}{\sqrt{6}}\left(\alpha_{1}^{3}+\gamma_{1}^{3}\right) \\
& +\frac{27}{\sqrt{6}}\left(\alpha_{1} \alpha_{2}^{2}+\gamma_{1} \gamma_{2}^{2}\right)-2|\breve{B}|^{2} \\
& -6|\breve{B}|^{4}-3|\breve{B}|^{2}\left(\alpha_{1}^{2}+\alpha_{2}^{2}+\gamma_{1}^{2}+\gamma_{2}^{2}\right) \\
& -\frac{9}{\sqrt{6}}\left(\alpha_{1}+\gamma_{1}\right)\left(2 \breve{b}_{3}^{2}-\left(\breve{b}_{1}^{2}+\breve{b}_{2}^{2}\right)\right) \\
& -\frac{9}{\sqrt{2}}\left(\alpha_{2}+\gamma_{2}\right)\left(\breve{b}_{2}^{2}-\breve{b}_{1}^{2}\right)+36 \breve{b}_{1} \breve{b}_{2} \breve{b}_{3},
\end{aligned}
$$

being negative on the semi-algebraic variety defined by the inequalities

$$
\sqrt{3} \alpha_{1} \leq \alpha_{2} \leq 0, \sqrt{3} \gamma_{1} \leq \gamma_{2} \leq 0,0 \leq \breve{b}_{1} \leq \breve{b}_{2} \leq \breve{b}_{3},
$$

and

$$
0<3\left(\alpha_{1}^{2}+\alpha_{2}^{2}+\gamma_{1}^{2}+\gamma_{2}^{2}\right)+6 \sum_{i=1}^{3} \breve{b}_{i}^{3}<2 .
$$

\section{B. Dependence on the distorsion in the lower two $\widehat{Z}^{2}$-eigenvalues.}

In this paragraph we assume that $b_{1}^{2}+b_{2}^{2}$ and all variables but $b_{1}$ and $b_{2}$ are fixed and produce a sharp upper bound for $S_{1}(R)$, independent of the ratio $b_{1} / b_{2}$.

Lemma 12. Eliminating the variable $b_{1} / b_{2}$ leads to the (sharp) upper bound $S_{2}(R)$ for the curvature polynomial $S_{1}(R)$, given as follows.

$$
\begin{aligned}
S_{1}(R) \leq & -3\left(\alpha_{1}^{2}+\alpha_{2}^{2}+\gamma_{1}^{2} \gamma_{2}^{2}\right)-\frac{9}{\sqrt{6}}\left(\alpha_{1}^{3}+\gamma_{1}^{3}\right)+\frac{27}{\sqrt{6}}\left(\alpha_{1} \alpha_{2}^{2}+\gamma_{1} \gamma_{2}^{2}\right) \\
& -2|\breve{B}|^{2}-6|\breve{B}|^{4}-3|\breve{B}|^{2}\left(\alpha_{1}^{2}+\alpha_{2}^{2}+\gamma_{1}^{2}+\gamma_{2}^{2}\right) \\
& -\frac{9}{\sqrt{6}}\left(\alpha_{1}+\gamma_{1}\right)\left(3 b_{3}^{2}-|\breve{B}|^{2}\right) \\
& +\frac{9}{\sqrt{2}}\left(|B|^{2}-\breve{b}_{3}^{2}\right)\left(\left(\alpha_{2}+\gamma_{2}\right)^{2}+8 \breve{b}_{3}^{2}\right)^{1 / 2} \\
= & S_{2}(R) .
\end{aligned}
$$


Proof. Let us introduce the auxiliary variable $x=\breve{b}_{1}^{2} /\left(\breve{b}_{1}^{2}+\breve{b}_{2}^{2}\right)$; then the constraint $0 \leq b_{1} \leq b_{2}$ may be written $x \in[0,1 / 2]$. The only term in $S_{1}(R)$ involving the ratio $b_{1} / b_{2}$ is

$$
9\left(-\left(\alpha_{2}+\gamma_{2}\right)\left(\breve{b}_{2}^{2}-\breve{b}_{1}^{2}\right)+4 \breve{b}_{1} \breve{b}_{2} \breve{b}_{3}\right) .
$$

Clearly enough, $\frac{\breve{b}_{1}^{2}+\breve{b}_{2}^{2}}{\sqrt{2}}\left(\left(\alpha_{2}+\gamma_{2}\right)^{2}+8 \breve{b}_{3}^{2}\right)^{\frac{1}{2}}$ is a sharp upper bound for the function

$$
f(x)=\left(\breve{b}_{1}^{2}+\breve{b}_{2}^{2}\right)\left(-\frac{\left(\alpha_{2}+\gamma_{2}\right)}{\sqrt{2}}(1-2 x)+4 \breve{b}_{3} \sqrt{x(1-x)}\right)
$$

on the interval $[0,1 / 2]$. This proves the lemma.

\section{Dependence on the largest $\widehat{Z}^{2}$-eigenvalue.}

Introduce two more auxiliary variables, $u=\alpha_{2}+\gamma_{2}$ and $v=a_{1}^{\prime}+\gamma_{1}$.

Lemma 13. The algebraic expression $S_{2}(R)$ admits the sharp upper bounds $S_{3}(R)$ and $s_{3}(R)$ which don't involve the ratio $\breve{b}_{3} /|\breve{B}|$.

When $|\breve{B}|^{2} \geq\left(3 v^{2}-u^{2}\right) / 8$, then

$$
\begin{aligned}
S_{2}(R) \leq & -3\left(1+|\breve{B}|^{2}\right)\left(\alpha_{1}^{2}+\alpha_{2}^{2}+\gamma_{1}^{2}+\gamma_{2}^{2}\right)-\frac{9}{\sqrt{6}}\left(\alpha_{1}^{3}+\gamma_{1}^{3}\right) \\
& +\frac{27}{\sqrt{6}}\left(\alpha_{1} \alpha_{2}^{2}+\gamma_{1} \gamma_{2}^{2}\right)-2|B|^{2}-6|\breve{B}|^{4}+\frac{3}{4 \sqrt{6}}\left(u^{2}+v^{2}+\varepsilon_{i}|\breve{B}|^{2}\right)^{3 / 2} \\
& +\frac{9}{4 \sqrt{6}}(-v)\left(\frac{v^{2}}{3}-u^{2}\right)=S_{3}(R) .
\end{aligned}
$$

When $|\breve{B}|^{2} \leq\left(3 v^{2}-u^{2}\right) / 8$, then

$$
\begin{aligned}
S_{2}(R) \leq & -3\left(\alpha_{1}^{2}+\alpha_{2}^{2}+\gamma_{1}^{2}+\gamma_{2}^{2}\right)\left(1+|B|^{2}\right)-\frac{9}{\sqrt{6}}\left(\alpha_{1}^{3}+\gamma_{1}^{3}\right) \\
& +\frac{27}{\sqrt{6}}\left(\alpha_{1} \alpha_{2}^{2}+\gamma_{1} \gamma_{2}^{2}\right)-2|\breve{B}|^{2}-6|\breve{B}|^{4} \\
& -\frac{18}{\sqrt{6}}\left(\alpha_{1}+\gamma_{1}\right)|\breve{B}|^{2}=s_{3}(R)
\end{aligned}
$$


Proof. The part of $S_{2}(R)$ involving the ratio $\breve{b}_{3} /|\breve{B}|$ is

$$
\frac{9}{\sqrt{2}}\left(-\left(3 \breve{b}_{3}^{2}-|\breve{B}|^{2}\right) \frac{\left(\alpha_{1}+\gamma_{1}\right)}{\sqrt{3}}+\left(|\breve{B}|^{2}-\breve{b}_{3}^{2}\right)\left(8 \breve{b}_{3}^{2}+\left(\alpha_{2}+\gamma_{2}\right)^{2}\right)^{1 / 2}\right) \text {. }
$$

On the interval $\left[\frac{|\breve{B}|^{2}}{3},|\breve{B}|^{2}\right]$, the first derivative of this expression w.r.t. the variable $b_{3}^{2}$ has the same sign as some quadratic polynomial in $b_{3}^{2}$. It should be clear that a sharp upper bound for the above expression in $\breve{b}_{3}^{2}$ on the interval $\left[\frac{|\breve{B}|^{2}}{3},|\breve{B}|^{2}\right]$ is given by its value

$$
\frac{1}{3}\left(|\breve{B}|^{2}+\frac{1}{4}\left(v^{2}-u^{2}+\left(\left(u^{2}-v^{2}\right)^{2}+8|\breve{B}|^{2} v^{2}+u^{2}\left(3 v^{2}-u^{2}\right)\right)^{1 / 2}\right)\right)
$$

at the critical point, provided this point belongs to the interval $\left[\frac{|B|^{2}}{3},|\breve{B}|^{2}\right]$. This is equivalent to the system

$$
\left\{\begin{array}{l}
8|\breve{B}|^{2}-v^{2}+u^{2} \geq 0 \\
64|\breve{B}|^{4}-8|\breve{B}|^{2}\left(3 v^{2}-2 u^{2}\right)-u^{2}\left(3 v^{2}-u^{2}\right) \geq 0,
\end{array}\right.
$$

which, in turn, is equivalent to the inequality $|\breve{B}|^{2} \geq \frac{3 v^{2}-u^{2}}{8}$.

If $|\breve{B}|^{2}<\frac{3 v^{2}-u^{2}}{8}$, then an upper bound for the former expression in $\breve{b}_{3}^{2}$, on the interval $\left[\frac{|\breve{B}|^{2}}{3},|\breve{B}|^{2}\right]$, is provided by its value at $|\breve{B}|^{2}$. After a straightforward substitution and some algebraic eliminations we obtain the upper bound (13). This is a sharp upper bound for the fundamental polynomial which involves only the norm of the trace-free Ricci curvature without referring any longer to the explicit spectrum, nor to any coupling with the Weyl curvature.

\section{Isolating $S^{1} \times S^{3}$-type curvatures.}

This part is devoted to a proof of the algebraic reduction for the case $|\breve{B}|^{2} \geq\left(3 v^{2}-u^{2}\right) / 8$.

Proposition 14. Under the assumption $8|\breve{B}|^{2} \geq 3 v^{2}-u^{2}$ the polynomial $S_{3}(R)$ is non-positive on the semi-algebraic variety defined by $\sqrt{3} \alpha_{1} \leq \alpha_{2} \leq$ $0, \sqrt{3} \gamma_{1} \leq \gamma_{2} \leq 0$ and $0<3\left(\alpha_{1}^{2}+\alpha_{2}^{2}+\gamma_{1}^{2}+\gamma_{2}^{2}\right)+6|\breve{B}|^{2}<2$. If it vanishes, either the curvature is of type $S^{4}$ (i.e. $\mathcal{D} \equiv 0$ ) or of type $S^{1} \times S^{3}$ (i.e. $W \equiv 0$ and $\widehat{Z}^{2}=\frac{\mathrm{scal}^{2}}{36} \operatorname{id}_{\Lambda^{2}}$ ). 


\section{A. Eliminating some dependence on the spectrum of the conformal curvature.}

We introduce the variables $x=\alpha_{1}-\gamma_{1}$ and $y=\alpha_{2}-\gamma_{2}$, and thus obtain a system $(u, v, x, y)$ with respect to which the fundamental polynomial shows more symmetries. We start with a (sharp) upper bound which cancels some dependence on the spectrum of the Weyl curvature.

Lemma 15. The algebraic expression $S_{3}(R)$ admits the following sharp upper bound, $S_{4}(R)$, which no longer involves the ratio $x / y$.

$$
\begin{aligned}
S_{3}(R) \leq & -\frac{3}{2}\left(u^{2}+v^{2}+x^{2}+y^{2}\right)-\frac{9}{4 \sqrt{6}}\left((-v)\left(3 u^{2}-v^{2}\right)\right. \\
& \left.-3\left(x^{2}+y^{2}\right)\left(u^{2}+v^{2}\right)^{1 / 2}\right)-2|\breve{B}|^{2}-6|\breve{B}|^{4} \\
& -\frac{3}{2}|\breve{B}|^{2}\left(u^{2}+v^{2}+x^{2}+y^{2}\right)+\frac{3}{4 \sqrt{6}}\left(u^{2}+v^{2}+8|\breve{B}|^{2}\right)^{3 / 2} \\
& +\frac{9}{4 \sqrt{6}}(-v)\left(v^{2}-u^{2}\right)-\frac{3}{2 \sqrt{6}}(-v) v^{2}=S_{4}(R) .
\end{aligned}
$$

Proof. The only terms involving the ratio $x / y$ in $S_{3}(R)$ are, up to a factor of $\frac{9}{\sqrt{6}}$,

$$
-\left(\alpha_{1}^{3}+\gamma_{1}^{3}\right)+3\left(\alpha_{1} \alpha_{2}^{2}+\gamma_{1} \gamma_{2}^{2}\right)
$$

Switching to the $x, y, u$ and $v$ variables this can be rewritten

$$
-\frac{1}{4}\left(v^{3}-3 u^{2} v\right)-\frac{3}{4} v\left(x^{2}-y^{2}-2 u x y\right)
$$

Introducing the auxiliary variable $\theta \in[0,2 \pi)$ defined by $x=\sqrt{x^{2}+y^{2}} \cos \theta$, and $y=\sqrt{x^{2}+y^{2}} \sin \theta$, one obtains,

$$
-\frac{1}{4}\left(v^{3}-3 u^{2} v\right)-\frac{3}{4}\left(x^{2}+y^{2}\right)(v \cos 2 \theta-u \sin 2 \theta) \text {. }
$$

As a function in $\theta$, this expression is $\pi$-periodic and achieves its maximum, which is equal to

$$
-\frac{1}{4}\left(v^{3}-3 u^{2} v\right)+\frac{3}{4}\left(x^{2}+y^{2}\right) \sqrt{u^{2}+v^{2}}
$$


at the points on the circle characterized by the equation $\operatorname{tg} 2 \theta_{0}=-u v^{-1}$ and $\theta_{0} \in\left[\frac{3 \pi}{4}, \pi\right] \cup\left[\frac{7 \pi}{4}, 2 \pi\right]$. This proves the lemma.

\section{B. Reducing the dependence of the upper bound on the conformal curvature to the variables $u$ and $v$ and eliminating the Ricci curvature.}

The previous bound $S_{4}(R)$ is clearly monotonic w.r.t. $\left(x^{2}+y^{2}\right)$, so that we are in either of the following two cases:

1. $S_{4}(R)$ is decreasing w.r.t. $\left(x^{2}+y^{2}\right)$ : the case $1+|\breve{B}|^{2} \geq$ $\frac{9}{2 \sqrt{6}}\left(u^{2}+v^{2}\right)^{1 / 2}$.

Proposition 16. If $1+|\breve{B}|^{2} \geq \frac{9}{2 \sqrt{6}}\left(u^{2}+v^{2}\right)^{1 / 2}$, then the upper bound $S_{4}(R)$ is non-positive on the semi-algebraic variety defined by $\sqrt{3} \alpha_{1} \leq \alpha_{2} \leq$ $0, \sqrt{3} \gamma_{1} \leq \gamma_{2} \leq 0$ and $0<3\left(\alpha_{1}^{2}+\alpha_{2}^{2}+\gamma_{1}^{2}+\gamma_{2}^{2}\right)+6|\breve{B}|^{2}<2$ and zero only for curvature with $\mathcal{D} \equiv 0$ ( $S^{4}$-type), or $W \equiv 0$ and $\widehat{Z}^{2}=\frac{\text { scal }^{2}}{36} \operatorname{id}_{\Lambda^{2}}$.

Proof. Under the assumption $1+|\breve{B}|^{2} \geq \frac{9}{2 \sqrt{6}}\left(u^{2}+v^{2}\right)^{\frac{1}{2}}$, the value of $S_{4}(R)$ on $x^{2}+y^{2}=0$ provides a (sharp) upper bound for $S_{4}(R)$, which is written

$$
\begin{aligned}
S_{5}(R)= & -\frac{3}{2}\left(u^{2}+v^{2}\right)-\frac{9}{4 \sqrt{6}}(-v)\left(3 u^{2}-v^{2}\right)-2|\breve{B}|^{2}-6|\breve{B}|^{4} \\
& -\frac{3}{2}|\breve{B}|^{2}\left(u^{2}+v^{2}\right)+\frac{3}{4 \sqrt{6}}\left(u^{2}+v^{2}+8|\breve{B}|^{2}\right)^{3 / 2} \\
& +\frac{9}{4 \sqrt{6}}(-v)\left(v^{2}-u^{2}\right)-\frac{3}{2 \sqrt{6}}(-v) v^{2} .
\end{aligned}
$$

Introducing the variable $X=\left(8|\breve{B}|^{2}+u^{2}+v^{2}\right)^{1 / 2}$, the derivative w.r.t. $|\breve{B}|^{2}$ is

$$
-\frac{3}{2} X^{2}+\frac{3 \sqrt{6}}{2} X-2 \text {. }
$$

Therefore, either $(-v) \geq \frac{1}{\sqrt{6}}$, and the value at

$$
|\breve{B}|^{2}=|\breve{B}|_{\max }^{2}=\frac{1}{3}-\left(\frac{u^{2}+v^{2}}{4}\right)
$$


is a sharp upper bound for $S_{5}(R)$, or

$$
\frac{2}{\sqrt{6}} \in\left[2(-v),\left(\frac{8}{3}-\left(u^{2}+v^{2}\right)\right)^{1 / 2}\right]
$$

in which case $S_{5}(R)$ achieves its upper bound on the boundary of the interval

$$
\left[|B|_{\min }^{2}=\frac{3 v^{2}-u^{2}}{8},|\breve{B}|_{\max }^{2}=\frac{1}{3}-\left(\frac{u^{2}+v^{2}}{4}\right)\right] .
$$

This is because $X_{\max }=\left(\frac{8}{3}-\left(u^{2}+v^{2}\right)\right)^{1 / 2}$ is always larger than. $2 / \sqrt{6}$ since $u^{2}+v^{2} \leq \frac{4}{3}$ - and smaller than $4 / \sqrt{6}$.

When evaluating $S_{5}(R)$ at $|\breve{B}|^{2}=|\breve{B}|_{\max }^{2}$, one gets

$S_{6}(R)=-\frac{4}{3}-\frac{u^{2}+v^{2}}{2}+\frac{3}{4 \sqrt{6}}\left(\frac{8}{3}-\left(u^{2}+v^{2}\right)\right)^{3 / 2}+\frac{3}{\sqrt{6}}(-v)\left(-3 u^{2}+v^{2}\right)$

which we can consider as a function of the variables $u^{2}+v^{2}$ and $\frac{v^{2}}{u^{2}+v^{2}}$ : it is then clearly increasing w.r.t. the variable $v^{2} /\left(u^{2}+v^{2}\right)$. Moreover, the bound $5 v^{2}+u^{2} \leq \frac{8}{3}$ is automatically satisfied if $u^{2}+v^{2} \leq \frac{8}{15}$. This leads to the following sharp upper bound for $S_{6}(R)$, not depending on $v^{2} /\left(u^{2}+v^{2}\right)$. Set $k=u^{2}+v^{2}$ :

$$
S_{6}(R) \leq-\frac{4}{3}-\frac{k}{2}+\frac{3}{4 \sqrt{6}}\left(\frac{8}{3}-k\right)^{3 / 2}+\frac{3}{\sqrt{6}} k^{3 / 2} .
$$

This last expression can be checked to be convex in $k$. It has value $\frac{8}{5}\left(\frac{2}{\sqrt{5}}-1\right)<0$ at $k=8 / 15$, and 0 at $k=0$. This non-negative value for the upper bound corresponds to $u=v=0=x=y$ and $|\breve{B}|^{2}=|\breve{B}|_{\max }^{2}=1 / 3$; moreover, in this case, $\alpha_{i}=\gamma_{i}=0$ for $i \in\{1,2\}$, and the critical value for $b_{3}^{2}$ (see (14)) is $\frac{|\breve{B}|^{2}}{3}=\frac{1}{9}$, which, in turn, implies $\breve{b}_{1}^{2}=\breve{b}_{2}^{2}=\frac{|\breve{B}|^{2}}{3}=\frac{1}{9}$. Going back to the classical geometric invariants these identities can be rewritten $W=0$ and $\widehat{Z}^{2}=\frac{\text { scal }^{2}}{36} \mathrm{id}_{\Lambda^{2}}$. It will be checked later that this corresponds to $S^{1} \times S^{3}$-type curvatures.

In the case where $\frac{8}{15} \leq u^{2}+v^{2} \leq \frac{4}{3}$, only two arcs of the circle $u^{2}+v^{2}=k$ (those points on the circle satisfying $5 v^{2}+u^{2} \leq \frac{8}{3}$ ) need be considered so that the value at $u^{2}=\frac{5}{4} k-\frac{2}{3}$ and $v^{2}=-\frac{k}{4}+\frac{2}{3}$, which simplifies to

$$
-\left(\frac{4}{3}+\frac{k}{2}\right)+\frac{3}{4 \sqrt{6}}\left(\frac{8}{3}-k\right)^{1 / 2}(8-9 k)
$$


is the sharp upper bound for $S_{6}(R)$ we shall consider. This function is decreasing in $k$ on the interval $\left[\frac{8}{15}, \frac{4}{3}\right]$ and has value $\frac{8}{5}\left(\frac{2}{\sqrt{5}}-1\right)<0$ at $\frac{8}{15}$.

It remains to evaluate $S_{5}(R)$ at $|\breve{B}|^{2}=|\breve{B}|_{\min }^{2}$, which, as a function of $k=u^{2}+v^{2}$ and $v$ may be written

$$
\Sigma_{6}(R)=-\frac{18}{\sqrt{6}} v^{3}-v^{2}+\frac{9 k}{\sqrt{6}} v-\frac{3}{2} v^{4}-\frac{5}{4} k+\frac{3}{32} k^{2} .
$$

The second derivative of $\Sigma_{6}(R)$ w.r.t. $v$ is the quadratic polynomial

$$
-2\left(9 v^{2}+\frac{54}{\sqrt{6}} v+1\right) \text {. }
$$

Since $\sqrt{3} \alpha_{1} \leq \alpha_{2} \leq 0$ and $\sqrt{3} \gamma_{1} \leq \gamma_{2} \leq 0$, it follows that $u^{2} \leq 3 v^{2}$ and that $v$ belongs to the interval $\left[-k^{1 / 2},-\frac{k}{2}^{1 / 2}\right]$, a subinterval of $[-2 / \sqrt{(3)}, 0]$. From this, one can check the maximum of $\Sigma_{6}(R)$ in $v$ on the interval $\left[v_{\min }(k), v_{\max }(k)\right]$ is achieved on the boundary of the interval.

Note that either $k \leq 8 / 15$ and so $v_{\min }=-k^{1 / 2}$, or $k \in\left[\frac{8}{15}, \frac{4}{3}\right]$ in which case $v_{\min }=-\frac{1}{2}\left(\frac{8}{3}-k\right)^{1 / 2}$ (because of the relation $\left.5 v^{2}+u^{2} \leq \frac{8}{3}\right)$. As for the upper bound $v_{\max }=-\frac{k}{2}^{1 / 2}$, which corresponds to $v^{2}=\frac{k}{4}$ and $u^{2}=\frac{3 k}{4}$, the relation $5 v^{2}+u^{2} \leq \frac{8}{3}$ is automatically satisfied since $k \leq \frac{2}{3}\left(\breve{\Delta}_{r^{+}}+\breve{\Delta}_{r^{-}}\right) \leq$ $\frac{4}{3}$.

In the case where $k \leq \frac{8}{15}$, we have $v_{\min }=-\sqrt{k}$ (and $u=0$ ) so that

$$
\Sigma_{6}(R)_{\mid v=v_{\min }}=9 k\left(-\frac{1}{4}+\frac{\sqrt{k}}{6}-\frac{5}{32} k\right),
$$

which is negative on $[0,8 / 15]$.

In the case where $\frac{4}{3} \geq k>\frac{8}{15}$, we have $v_{\min }^{2}=\frac{2}{3}-\frac{k}{4}$ and

$$
\Sigma_{6}(R)_{\mid v^{2}=\frac{2}{3}-\frac{k}{4}}=\frac{9}{\sqrt{6}}\left(\frac{2}{3}-\frac{k}{4}\right)^{1 / 2}\left(\frac{4}{3}-\frac{3 k}{2}\right)-\frac{k}{2}-\frac{4}{3} ;
$$

this last expression can be shown to be decreasing and negative on the interval $\left[\frac{8}{15}, \frac{4}{3}\right]$.

As for the value at $v_{\max }=-\frac{\sqrt{k}}{2}$, one has

$$
\Sigma_{6}(R)_{\mid v=-\frac{\sqrt{k}}{2}}=-\frac{3 k}{2}\left(1+\frac{3}{2 \sqrt{6}} \sqrt{k}\right),
$$


which is non-positive $(k \geq 0)$ and zero only if $k=0$. In this latter case, $|\breve{B}|^{2}=|\breve{B}|_{\min }^{2}=\frac{3 v^{2}-u^{2}}{8}=0$ and $\breve{\Delta}_{r^{+}}+\breve{\Delta}_{r_{-}}=\frac{3 k}{2}=0$, which means $\mathcal{D} \equiv 0$ : this critical value of the upper bound corresponds here to curvatures of type $\left(S^{n}\right.$, can $)$.

2. $S_{4}(R)$ is increasing w.r.t. $\left(x^{2}+y^{2}\right)$ : the case $1+|\breve{B}|^{2} \leq$ $\frac{9}{2 \sqrt{6}}\left(u^{2}+v^{2}\right)^{1 / 2}$.

Proposition 17. If $1+|\breve{B}|^{2} \leq \frac{9}{2 \sqrt{6}}\left(u^{2}+v^{2}\right)^{1 / 2}$, then the upper bound $S_{4}(R)$ is negative.

Here the value on $x^{2}+y^{2}=\frac{2}{3}\left(2-6|\breve{B}|^{2}\right)-u^{2}-v^{2}$ provides the sharp upper bound for $S_{4}(R)$. Recall that, in this part, $|\breve{B}|^{2} \geq \frac{3 v^{2}-u^{2}}{8} \geq 0$, and note that the further assumption $1+|\breve{B}|^{2} \leq \frac{9}{2 \sqrt{6}}\left(u^{2}+v^{2}\right)^{1 / 2}$ implies $k \geq \frac{8}{27}\left(1+|\breve{B}|^{2}\right)^{2} \geq \frac{8}{27}$.

The value on $x^{2}+y^{2}=\frac{2}{3}\left(2-6|\breve{B}|^{2}\right)-u^{2}-v^{2}$ is

$$
\begin{gathered}
S_{4}(R) \leq-2+2|\breve{B}|^{2}+\frac{9}{\sqrt{6}}\left(1-3|\breve{B}|^{2}\right) \sqrt{k}+\frac{3}{4 \sqrt{6}}\left(k+8|\breve{B}|^{2}\right)^{3 / 2} \\
-\frac{27}{4 \sqrt{6}} k^{3 / 2}-\frac{9}{\sqrt{6}}(-v)\left(u^{2}-\frac{v^{2}}{3}\right)=T_{5}(R) .
\end{gathered}
$$

As a function of $|\breve{B}|^{2}$ this expression can be checked to be convex; in particular the values of $T_{5}(R)$ on $|\breve{B}|_{\min }^{2}=\frac{3 v^{2}-u^{2}}{8}$ and $|\breve{B}|_{\max }^{2}=\frac{1}{3}-\frac{k}{4}$ provide the upper bound we are looking for.

On $|B|^{2}=|B|_{\min }^{2}=\frac{3 v^{2}-u^{2}}{8}, T_{5}(R)$ evaluates to the following simple expression, using the variable $x=v^{2}-u^{2}$ :

$T_{6}(R)=-2+\frac{k}{4}+\frac{9}{\sqrt{6}} \sqrt{k}-\frac{81}{8 \sqrt{6}} k^{3 / 2}+\left(\frac{1}{2}-\frac{27}{4 \sqrt{6}} k^{1 / 2}\right) x+\frac{3 \sqrt{3}}{2} x(k+x)^{1 / 2}$.

This expression can be shown to be convex in $x$ on the interval $\left[-\frac{k}{2}, k\right]$ (note that $3 v^{2} \geq u^{2} \geq 0$ means that $\left.x \in\left[-\frac{k}{2}, k\right]\right)$ and has value

$$
-2+\frac{9}{\sqrt{6}} k^{1 / 2}-\frac{9}{\sqrt{6}} k^{3 / 2}
$$


at $x=-\frac{k}{2}$. This is negative for $k$ in the interval [8/27,4/3]. At $x=k$ it has value

$$
-2+\frac{9}{\sqrt{6}} \sqrt{k}+\frac{3}{4} k-\frac{63}{8 \sqrt{6}} k^{3 / 2}
$$

The function $-2+\frac{9}{\sqrt{6}} y+\frac{3}{4} y^{2}-\frac{63}{8 \sqrt{6}} y^{3}$ is concave on the interval $\left[-\frac{2 \sqrt{2}}{3 \sqrt{3}}, \frac{2}{\sqrt{3}}\right]$, and achieves its maximum at $y=\frac{2}{7} \sqrt{6}$, where it evaluates to $-8 / 49<0$.

On $|B|^{2}=|B|_{\max }^{2}=\frac{1}{3}-\left(u^{2}+v^{2}\right) / 4, T_{5}(R)$ evaluates to the following simple expression, using the auxiliary variables $k=u^{2}+v^{2}$ and $x=v^{2}-u^{2}$ :

$$
\Theta_{6}(R)=-\frac{4}{3}-\frac{k}{2}+\frac{3}{4 \sqrt{6}}\left(\frac{8}{3}-k\right)^{3 / 2}-\frac{\sqrt{3}}{2}(k-2 x)(k+x)^{1 / 2},
$$

where $x \in[-k / 2, k], k \in[8 / 27,4 / 3]$ and $x \leq \frac{4}{3}-\frac{3}{2} k$ (since $\frac{3 v^{2}-u^{2}}{8} \leq \frac{1}{3}-\frac{k}{4}$ ). Note that $-k / 2 \leq \frac{4}{3}-\frac{3}{2} k$, since $k \leq \frac{4}{3}$ and that $k \leq \frac{4}{3}-\frac{3}{2} k$ is equivalent to $k \leq \frac{8}{15}$.

In any case, the former expression for $\Theta_{6}(R)$ is monotonic non-decreasing in $x$ on $[-k / 2, k]$ and has value

$$
\frac{3}{\sqrt{6}} k^{3 / 2}+\frac{3}{4 \sqrt{6}}\left(\frac{8}{3}-k\right)^{3 / 2}-\frac{k}{2}-\frac{4}{3}
$$

at $k$. This function of $k$ is convex on $\left[0, \frac{8}{15}\right]$ and is equal to $\frac{8}{15}\left(\frac{2}{\sqrt{5}}-1\right)<0$ at $\frac{8}{15}$ and to $-\frac{8}{81}\left(13-\frac{16}{\sqrt{2}}\right)<0$ at $\frac{8}{27}$.

In the case where $k \geq 8 / 15, \Theta_{6}(R)$ is equal to

$$
\frac{4}{3}-\frac{k}{2}+\left(\frac{8}{3}-k\right)^{1 / 2}\left(\sqrt{6}-\frac{27 k}{\sqrt{6}}\right)
$$

at $\frac{4}{3}-\frac{3}{2} k$. This expression turns out to be convex and negative on the interval $[8 / 15,4 / 3]$.

\section{Isolating $\mathbb{P}^{2}$-type curvatures.}

We consider in this part the remaining case : $8|\widetilde{B}|^{2} \leq 3 v^{2}-u^{2}$.

Proposition 18. Under the assumption $8|B|^{2} \leq 3 v^{2}-u^{2}$, the polynomial $\widetilde{S}_{3}(R)$ is non-positive on the semi-algebraic variety defined by

$$
\sqrt{3} \alpha_{1} \leq \alpha_{2} \leq 0
$$




$$
\begin{aligned}
\sqrt{3} \gamma_{1} & \leq \gamma_{2} \leq 0 \text { and } \\
0 & <3\left(\alpha_{1}^{2}+\alpha_{2}^{2}+\gamma_{1}^{2}+\gamma_{2}^{2}\right)+6|B|^{2}<2 .
\end{aligned}
$$

If it vanishes then one of the following three cases holds:

(1) Either $\mathcal{D} \equiv 0$ ( $S^{4}$-type curvatures),

(2) or $Z \equiv 0, \operatorname{spec}\left(\widehat{R}_{\mid \Lambda^{+}}\right)=\{1,0,0\}$ and $\widehat{R}_{\mid \Lambda^{-}}=i d_{\mid \Lambda^{-}}\left(\mathbb{P}^{2}\right.$-type curvature),

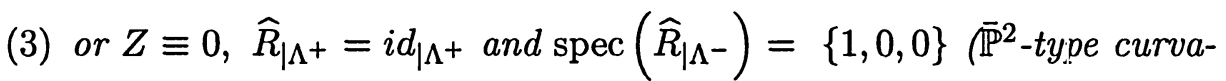
ture).

\section{A. Eliminating $x / y$ and $u / v$ to reduce the dependence on the conformal curvature.}

Recall that we have set $v=\alpha_{1}+\gamma_{1}$ and $u=\alpha_{2}+\gamma_{2}$. As in the previous paragraph let us introduce the variables $x=\alpha_{1}-\gamma_{1}$ and $y=\alpha_{2}-\gamma_{2}$ so that the only inhomogeneous factor in $s_{3}(R)$,

$$
-\frac{9}{\sqrt{6}}\left(\alpha_{1}^{3}+\gamma_{1}^{3}\right)+\frac{27}{\sqrt{6}}\left(\alpha_{1} \alpha_{2}^{2}+\gamma_{1} \gamma_{2}^{2}\right)
$$

may be written

$$
-\frac{9}{4 \sqrt{6}}\left(v^{3}-3 u^{2} v+3 v\left(x^{2}-y^{2}\right)-6 u x y\right) .
$$

In this expression the variable $x / y$ can be eliminated -as we have done in Lemma 15- to achieve the sharp upper bound

$$
\begin{aligned}
s_{3}(R) \leq- & \frac{3}{2}\left(x^{2}+y^{2}+k\right)\left(1+|\breve{B}|^{2}\right)+\frac{9}{4 \sqrt{6}}(-v)\left(4 v^{2}-3 k+3|B|^{2}\right) \\
& +\frac{27}{4 \sqrt{6}} k^{1 / 2}\left(x^{2}+y^{2}\right)-2|\breve{B}|^{2}-6|\breve{B}|^{4}=s_{4}(R)
\end{aligned}
$$

where we have set $k=u^{2}+v^{2}$.

Since $4 v^{2}-k=3 v^{2}-u^{2} \geq 0$, the expression for $s_{4}(R)$ is clearly monotonic non-increasing in $v$, when the variables $x^{2}+y^{2}, k$ and $|\breve{B}|^{2}$ are kept fixed. 
Eliminating the variable $v$ by substituting $v_{\min }=-k^{1 / 2}$ for $v$ leads to the following sharp upper bound

$$
\begin{gathered}
s_{5}(R)=-\frac{3}{2}\left(1+|\breve{B}|^{2}\right)\left(x^{2}+y^{2}+k\right)-\frac{9}{2 \sqrt{6}}\left(k^{3 / 2}-\frac{3 k^{1 / 2}}{2}\left(x^{2}+y^{2}+k\right)\right) \\
-2|\breve{B}|^{2}-6|\breve{B}|^{4}+\frac{18}{\sqrt{6}}|\breve{B}|^{2} k^{1 / 2} .
\end{gathered}
$$

\section{B. Eliminating $k$ to get a bound depending only on} the Ricci curvature. The case: $1+|\breve{B}|^{2} \geq \frac{9}{2 \sqrt{6}} k^{1 / 2}$.

If $\frac{9}{2 \sqrt{6}} k^{1 / 2} \leq 1+|\breve{B}|^{2}$, then $s_{5}(R)$ is non-increasing as a function of the variable $\left(x^{2}+y^{2}\right)$, so that we get an upper bound by setting $x^{2}+y^{2}=0$ in the above expression :

$s_{6}(R)=-2|\breve{B}|^{2}-6|\breve{B}|^{4}+\frac{3}{2}|\breve{B}|^{2} k^{1 / 2}\left(2 \sqrt{6}-k^{1 / 2}\right)+\frac{3}{2} k\left(\frac{3}{2 \sqrt{6}} k^{1 / 2}-1\right)$.

The assumption $8|B|^{2} \leq 3 v^{2}-u^{2}$ implies $k \geq \frac{8|B|^{2}}{3}$; the pinching assumption $\breve{\Delta}_{r^{+}}+\breve{\Delta}_{r^{-}}+6|\breve{B}|^{2} \leq 2$ amounts to $k \leq \frac{4}{3}-4|\breve{B}|^{2}$. Note that such a real $k$ exists only if $|B|^{2} \leq 1 / 5$.

The expression for $s_{6}(R)$ can be checked to be convex in $k$ on the interval $\left[\frac{8|\breve{B}|^{2}}{3}, \frac{4}{3}-4|\breve{B}|^{2}\right]$. Substituting $k=\frac{8|\breve{B}|^{2}}{3}$ into $s_{6}(R)$ one obtains $2|\breve{B}|^{2}\left(-3+8|\breve{B}|-5|\breve{B}|^{2}\right)$, which is negative on the interval $(0,1 / 5]$. Let us remark that the case $|\breve{B}|^{2}=0$ corresponds to $k=x^{2}+y^{2}=0$, i.e. $|\mathcal{D}|^{2}=0$ : this critical case is associated with curvatures of type $S^{4}$. Substituting $k=4\left(\frac{1}{3}-|\breve{B}|^{2}\right)$ into $s_{6}(R)$, one obtains

$$
-2+2|\breve{B}|^{2}+\frac{18}{\sqrt{6}}\left(\frac{1}{3}-|\breve{B}|^{2}\right)^{1 / 2}\left(\frac{1}{3}+|\breve{B}|^{2}\right) .
$$

This can be shown to be concave in $|\breve{B}|^{2}$ on $(0,1 / 3)$, and negative (since it is negative at the root of the derivative). 


\section{Eliminating $|\breve{B}|$ to get a bound depending only on the conformal curvature . \\ The case : $1+|\breve{B}|^{2} \leq \frac{9}{2 \sqrt{6}} k^{1 / 2}$.}

If $\frac{9}{2 \sqrt{6}} k^{1 / 2} \geq 1+|\breve{B}|^{2}$, then $s_{5}(R)$ is non-decreasing as a function of the variable $x^{2}+y^{2}$; the substitution $x^{2}+y^{2}=\frac{4}{3}-4|B|^{2}-u^{2}-v^{2}$ leads to the following upper bound

$$
s_{5}(R) \leq-2-|\breve{B}|^{2}\left(\frac{9}{\sqrt{6}} k^{1 / 2}-2\right)+\frac{9}{\sqrt{6}} k^{1 / 2}(1-k / 2) .
$$

Under the local assumption $\frac{9}{\sqrt{6}} k^{1 / 2} \geq 2+2|B|^{2} \geq 2$, one obtains the upper bound

$$
s_{5}(R) \leq-2+\frac{9}{\sqrt{6}} k^{1 / 2}(1-k / 2) .
$$

As a function of the variable $k^{1 / 2}$, the last expression is concave, non-positive and vanishes only for $k=2 / 3$.

If $s_{5}(R)$ also vanishes, we must have $|\breve{B}|^{2}=0$ and $x^{2}+y^{2}=2 / 3$; if $s_{4}(R)$ vanishes then $u=0$ and $v^{2}=k$ (cf. paragraph $\mathrm{V}-\mathrm{A}$ ).

If $s_{3}(R)$ also vanishes then, in Lemma $15, \theta_{0} \in\{0, \pi\}$, i.e. $x \in$ $\left\{ \pm \sqrt{x^{2}+y^{2}}\right\}=\left\{ \pm \sqrt{\frac{2}{3}}\right\}$ and $y=0$.

Let us assume that $8|\breve{B}|^{2} \leq 3 v^{2}-u^{2}$ and $1+|\breve{B}|^{2} \leq \frac{9}{2 \sqrt{6}} k^{1 / 2}$; we have just proven that the fundamental polynomial cannot vanish except if $\alpha_{1}=-\sqrt{\frac{2}{3}}, \gamma_{1}=0, \alpha_{2}=0, \gamma_{2}=0$ and $|B|=0$, (which is equivalent to $|B|=0, \bar{a}_{1}=-1 / 3, \bar{a}_{2}=-1 / 3, \bar{a}_{3}=2 / 3$ and $\left.\bar{c}_{i}=0, i \in\{1,2,3\}\right)$, or if $\alpha_{1}=0, \gamma_{1}=-\sqrt{\frac{2}{3}}, \alpha_{2}=0, \gamma_{2}=0$ and $|B|=0$, (which is equivalent to $|B|=0, \bar{a}_{i}=0, i \in\{1,2,3\}$ and $\left.\bar{c}_{1}=\bar{c}_{2}=-\frac{1}{3}, \bar{c}_{3}=\frac{2}{3}\right)$. This means that $\widehat{R}$ preserves the Hodge splitting of $\Lambda^{2}$ and either $\operatorname{spec}\left(\widehat{R}_{\mid \Lambda^{+}}\right)=$ $\{1,0,0\}, \widehat{R}_{\mid \Lambda^{-}}=1 / 3$ id or $\widehat{R}_{\mid \Lambda^{+}}=1 / 3$ id and $\operatorname{spec}\left(\widehat{R}_{\mid \Lambda^{-}}\right)=\{1,0,0\}$, which corresponds to the curvature operator of $\left(\mathbb{P}_{2}, \mathrm{~F}-\mathrm{S}\right)$, respectively $\left(\overline{\mathbb{P}}_{2}\right.$, F-S).

This ends the proof of Proposition 18. It means that we have solved the algebraic reduction - and so we have proved Proposition 4. 


\section{End of the proof.}

Heuristically, at least, the remainder of the proof should now be clear : to get convergence to constant curvature along an integral curve from the monotonicity of $|\mathcal{D}|^{2} \mathrm{scal}^{-\beta}, \beta<2$, it basically remains to prove that not only the supremum of the curvature norm (see Proposition 1 ) but also that the infimum of the scalar curvature actually diverges at the first singularity developped by the flow. Technical details are very close to the arguments in [M1], which, in turn are an extension to higher dimensions of Hamilton's original argument for 3-manifolds. For the sake of completeness we provide them here.

\section{A. Gradient of the scalar curvature along an integral curve for the Ricci field.}

Proposition 19. For any 1/6-weakly pinched 4-manifold of positive scalar curvature $\left(V, g_{0}\right)$ and any positive real number $\eta$ there is a constant $C(\eta)$ s.t. along any integral curve of the Ricci field -2 ric originating from $g_{0}$, the gradient of the scalar curvature admits the following upper bound

$$
\mid d \text { scal } \mid<\eta \mathrm{scal}^{3 / 2}+C(\eta) .
$$

To start with, we derive the differential equation for $\mid d$ scal $\mid$ along an integral curve of the Ricci curvature :

Lemma 20. Along any integral curve of the Ricci field -2 ric the gradient of the scalar curvature satisfies the following differential identities :

$$
\left(\frac{\partial}{\partial t}+D^{*} D\right) \mid d \text { scal }\left.\right|^{2}=-2 \mid D d \text { scal }\left.\right|^{2}+4\left(d \mid \text { ric }\left.\right|^{2}, d \text { scal }\right)
$$

and,

$$
\begin{aligned}
\left(\frac{\partial}{\partial t}+D^{*} D\right) \mid d \mathrm{scal}^{2} \mathrm{scal}^{-1}= & 4 \mathrm{scal}^{-2}(D d \mathrm{scal}, d \mathrm{scal} \otimes d \mathrm{scal}) \\
& -\left.2\left|d \mathrm{scal}^{4} \mathrm{scal}^{-3}-2 \mathrm{scal}^{-1}\right| D d \mathrm{scal}\right|^{2} \\
& +4 \mathrm{scal}^{-1}\left(d|\mathrm{ric}|^{2}, d \mathrm{scal}\right) \\
& -\left.2\left|d \mathrm{scal}^{2}\right| \mathrm{ric}\right|^{2} \mathrm{scal}^{-2}
\end{aligned}
$$


Proof. Starting with identity (3), one can obtain $\frac{\partial}{\partial t} \mid d$ scal $\left.\right|^{2}=2(d$ scal $\otimes d$ scal, ric $)+2\left(d\left(-D^{*} D\right.\right.$ scal $+2 \mid$ ric $\left.\left.\right|^{2}\right), d$ scal $)$, and,

$$
D^{*} D \mid d \text { scal }\left.\right|^{2}=2\left(D^{*} D d \text { scal, } d \text { scal }\right)-2 \mid D d \text { scal }\left.\right|^{2}
$$

where

$$
D^{*} D d \text { scal }=-\sum_{i=1}^{\operatorname{dim} M=n}\left(D^{2} d \text { scal }\right)\left(X_{i}, X_{i}, \cdot\right)
$$

for some orthonormal moving frame $\left(X_{i}\right)_{i=1}^{n}$ on $M$. Because of the symmetry of the Hessian and the definition of curvature as a commutator,

$$
D^{*} D d \mathrm{scal}=-\sum_{i=1}^{n}\left(D(D d \mathrm{scal})\left(\cdot, X_{i}, X_{i}\right)+\left(R\left(\cdot, X_{i}\right) d \mathrm{scal}\right)\left(X_{i}\right)\right),
$$

i.e.

$$
D^{*} D d \mathrm{scal}=+d \Delta \mathrm{scal}+d \mathrm{scal}\left(\sum_{i=1}^{n} R\left(\cdot, X_{i}\right) X_{i}\right),
$$

which proves (15).

Identity (16) is then a routine corollary of the previous calculation.

Let us go back to the proof of Proposition 19 . The terms involving the covariant derivative of the Ricci curvature prohibit a straight:forward invoking of the maximum principle. In order to balance these terms we introduce a $|z|^{2}$-term, which we know to behave well under the flow:

Proposition 21. Let $\eta$ be some real number in the interval $(0,1 / 4)$. The curvature polynomial

$$
Q=|d \mathrm{scal}|^{2} \mathrm{scal}^{-1}+80|z|^{2}-\eta \mathrm{scal}^{2}
$$

is "almost" monotonic along the integral curve of the Ricci field originating from any $C-(C<1 / 6)$ weakly pinched metric of positive scalar curvature, in the sense that it satisfies the following differential equation

$$
\left(\frac{\partial}{\partial t}+\Delta\right) Q \leq C(\eta)
$$


Note. This is the 4-dimensional version of a general statement holding true in any dimension : see Lemma 83 in [M3 - Chap. 3].

Proof. From the evolution equation for the Ricci curvature (see (2)) and the scalar curvature (see (3)), one gets the following equation for the norm of the trace-free Ricci curvature

$$
\begin{aligned}
\left(\frac{\partial}{\partial t}+D^{*} D\right)|z|^{2}= & -2 \mid D \text { ric }\left.\right|^{2}+\frac{2}{n} \mid d \text { scal }\left.\right|^{2} \\
& +4(\stackrel{\circ}{R} \text { (ric), ric })-\frac{4}{n} \text { scal } \mid \text { ric }\left.\right|^{2} .
\end{aligned}
$$

A main step towards the previous proposition is an improvement on the trivial inequality $\frac{\mid d \text { scal }\left.\right|^{2}}{n} \leq \mid D$ ric $\left.\right|^{2}$, which relies on the study of a further splitting of the tensor field $D$ ric.

Lemma 22. Because of the invariance relation associated to the second Bianchi identity, we have the following stronger lower bound for $\mid D$ ric $\mid$

$$
\mid D \text { ric }\left.\right|^{2} \geq \frac{5}{18} \mid d \text { scal }\left.\right|^{2}
$$

instead of the silly $|D T|^{2} \geq \frac{1}{4}|d \operatorname{tr} T|^{2}$.

Proof. Let us write $D$ ric as a sum of a tensor $T_{1}$, all of whose traces vanish, and a tensor $T_{2}$, which is the (orthogonal) projection of $D$ ric on

$$
\operatorname{span}(d \operatorname{scal} \otimes g, g \otimes \delta \text { ric },(g \otimes \delta \text { ric }) \circ \sigma),
$$

where $\sigma$ is the 3-permutation $(3,1,2)$. By contracting the second Bianchi identity one obtains $\delta$ ric $=-\frac{1}{2} d$ scal and the symmetry of $D$ ric w.r.t. the last 2 variables then implies that there are some real numbers $a$ and $b$ s.t.

$$
T_{1}=a d \text { scal } \otimes g+b\left((d \text { scal } \otimes g) \circ \sigma+(d \text { scal } \otimes g) \circ \sigma^{2}\right) .
$$

These $a$ and $b$ are determined by considering all possible traces, which leads to the following system

$$
\left\{\begin{array}{l}
d \text { scal }=(4 a+2 b) d \text { scal } \\
-\delta \text { ric }=\frac{1}{2} d \text { scal }=(a+5 b) d \text { scal }
\end{array}\right.
$$


whose only solution is $a=\frac{2}{9}$ and $b=\frac{1}{18}$.

From the following identities

$(d$ scal $\otimes g, d$ scal $\otimes g \circ \sigma)=\left(d\right.$ scal $\otimes g, d$ scal $\left.\otimes g \circ \sigma^{2}\right)$

$$
=\left(d \text { scal } \otimes g \circ \sigma, d \text { scal } \otimes g \circ \sigma^{2}\right)=\mid d \text { scal }\left.\right|^{2},
$$

we then deduce the lower bound we have claimed :

$$
\mid D \text { ric }\left.\right|^{2}=\left|T_{1}\right|^{2}+\left|T_{2}\right|^{2} \geq\left(4\left(a^{2}+2 b^{2}\right)+4 a b+2 b^{2}\right) \mid d \text { scal }\left.\right|^{2} .
$$

Back to the proof of Proposition 19, a straightforward corollary of the previous lemma is the following differential inequality which holds along any integral curve of the Ricci field -2 ric

$$
\left(\frac{\partial}{\partial t}+D^{*} D\right)|z|^{2} \leq-\frac{1}{5} \mid D \text { ric }\left.\right|^{2}+4(\stackrel{\circ}{R} \text { (ric), ric })-\text { scal } \mid \text { ric }\left.\right|^{2} .
$$

The next step consists in evaluating the curvature polynomial ( $\stackrel{\circ}{R}$ (ric), ric).

Lemma 23. For any Riemannian manifold $(M, g)$ which is more pinched than $S^{1} \times\left(S^{3}\right.$, can) and any positive real numbers $k$ and $\eta$, there is some constant $C=C(\eta, k, g)$ such that the following inequality holds

$$
k(\stackrel{\circ}{R} \text { (ric), ric })-\frac{\text { scal }}{4}|\mathrm{ric}|^{2}<\eta \mathrm{scal}^{3}+C .
$$

Proof. Because of the identities

$$
\begin{aligned}
& z \stackrel{\circ}{\circledast} g(z)=|z|^{2} g-2 z \circ z, \\
& g \stackrel{\circledast}{\circledast} g(g)=6 g, \\
& g \stackrel{\circledast}{\circledast} g(z)=-2 z \quad \text { and } \\
& z \circledast g(g)=2 z,
\end{aligned}
$$

the curvature polynomial $(\stackrel{\circ}{R}$ (ric), ric), can be written

$$
(\stackrel{\circ}{R} \text { (ric), ric })=\frac{\mathrm{scal}^{3}}{16}+\frac{5}{12} \mathrm{scal}|z|^{2}-\operatorname{tr} z^{3}+(\stackrel{\circ}{W}(z), z)
$$




$$
\leq \frac{\text { scal }}{4}|\mathrm{ric}|^{2}+\frac{\text { scal }}{6}|z|^{2}+|z|^{3}+|W||z|^{2} .
$$

Theorem 1 then implies that there is some real number $\beta_{0}$ in the interval $[1,2)$ s.t. $|\mathcal{D}|^{2} \mathrm{scal}^{-\beta}$ is uniformly bounded (since it is monotonic) on the integral curve issued by $g$, provided $\beta \in\left[\beta_{0}, 2\right]$. In particular there exists some real number $K$ depending only on $g$ s.t.

$$
(\stackrel{\circ}{R} \text { (ric), ric })-\frac{\text { scal }}{4}|\mathrm{ric}|^{2} \leq K\left(\text { scal }^{1+\beta_{0}}+\mathrm{scal}^{\frac{3 \beta_{0}}{2}}\right) .
$$

Define

$$
c=c\left(\eta ; k, K, \beta_{0}\right)=\sup _{x \in \mathbb{R}^{+}} k K\left(x^{1+\beta_{0}}+x^{\frac{3 \beta_{0}}{2}}\right)-\eta x^{3}(<+\infty) .
$$

Then

$$
k\left((\stackrel{\circ}{R} \text { (ric), ric })-\frac{\text { scal }}{4}|\mathrm{ric}|^{2}\right) \leq C\left(n, k, K, \beta_{0}\right)+\eta \mathrm{scal}^{3},
$$

which proves the lemma.

Using the identities (3), (15) and (16) and the inequality (14) we can derive the following evolution equation for the curvature polynomial $Q$

$$
\begin{aligned}
\left(\frac{\partial}{\partial t}+D^{*} D\right) Q \leq & -2 \mathrm{scal}^{-3}|d \mathrm{scal} \otimes d \mathrm{scal}-\mathrm{scal} D d \mathrm{scal}|^{2} \\
& +4 \mathrm{scal}^{-1}\left(d|\mathrm{ric}|^{2}, d \mathrm{scal}\right) \\
& -\left.2\left|d \mathrm{scal}^{2}\right| \mathrm{ric}\right|^{2} \mathrm{scal}^{-2}-16|D \mathrm{ric}|^{2} \\
& +320\left((\stackrel{\circ}{R} \text { (ric) }, \text { ric })-\frac{\mathrm{scal}}{4}|\mathrm{ric}|^{2}\right) \\
& +2 \eta|d \mathrm{scal}|^{2}-4 \eta \text { scal |ric }\left.\right|^{2}
\end{aligned}
$$

We can now observe that $1 / 6-(C-, C<1 / 6)$ weak pinching implies that the Ricci curvature is non-negative (resp. positive). That this statement is sharp can be checked on the product $S^{1} \times\left(S^{3}\right.$, can $)$.

Lemma 24. Any 1/6-weakly pinched $(C-, C<1 / 6$, weakly pinched) Riemannian 4-manifold of positive scalar curvature has non-negative (resp. positive) Ricci curvature. 
Proof. First observe the trivial bound $|z|^{2}=\frac{1}{2}|Z|^{2} \leq \frac{1}{2}|\mathcal{D}|^{2} \leq \frac{C}{2}$ scal $^{2}$. Since $z$ is trace free (by definition), a sharp lower bound for the $z$-spectrum is $-\frac{1}{2} \sqrt{\frac{3}{2}} \sqrt{C}$ scal, which is not less than $-\frac{\text { scal }}{4}$ (resp. greater than). Since it is equal to $z+\frac{\text { scal }}{4} g$, the Ricci curvature is non-negative (resp. positive). One way to get this lower bound for the $z$-spectrum is to note that the critical set for the $x_{1}$-function on the intersection of the hypersurfaces $\sum_{i=1}^{n} x_{i}=0$ and $\sum_{i=1}^{n} x_{i}^{2}=C$ is characterized by the existence of two Lagrange multipliers $\lambda$ and $\mu$ s.t.

$$
\left(1-\lambda x_{1}-\mu\right) d x_{1}-\sum_{i \geq 2}\left(\lambda x_{i}+\mu\right) d x_{i}=0 .
$$

As a result, all coordinates but $x_{1}$ are equal : one can then check that either $x_{1}=\sqrt{\frac{C(n-1)}{n}}$ (maximum) or $x_{1}=-\sqrt{\frac{C(n-1)}{n}}$ (minimum).

Let $\mathrm{C}$ be less than or equal to $1 / 6$. By Proposition 4, $C$-weak pinching is preserved by the flow. The previous lemma then implies that the Ricci curvature is non-negative (and positive if $C$ is less than 1/6) along the integral curve originating from a $C$ - weakly pinched manifold. Hence we can use the trace to get $\mathrm{scal}^{2}$ as an upper-bound for the square of the norm of the Ricci curvature , $|\mathrm{ric}|^{2}$, along any integral curve of the field -2 ric starting at a $C-\left(\leq \frac{1}{6}\right)$ weakly pinched metric. As a consequence, Lemma 23 and the differential inequality (18) imply the simpler differential inequality for the (differential) curvature polynomial $Q$

$$
\left(\frac{\partial}{\partial t}+D^{*} D\right) Q \leq C(\eta, 320, g)
$$

provided $\eta \in(0,1 / 4)$; here $C$ is the constant in Lemma 23. This ends the proof of Proposition 21.

Corollary 25. Associated to any $C\left(<\frac{1}{6}\right)$ weakly pinched 4-mainifold of positive scalar curvature $\left(M, g_{0}\right)$ and any real number in the interval $(0,1 / 4)$ there is some constant $C\left(\nu, g_{0}\right)$ s.t. along the integral curve of the Ficci curvature, originating from the metric $g_{0}$, the gradient of the scalar curvature is uniformly bounded by the scalar curvature as follows

$$
\mid d \text { scal } \mid<\nu \mathrm{scal}^{3 / 2}+C\left(\nu, g_{0}\right) \text {. }
$$

Proof. First note that the evolution equation for the scalar curvature (3) and the (parabolic) maximum principle lead to a (finite) upper bound for 
the length of any integral curve of the field -2 ric in terms of a pointwise lower bound for the scalar curvature of the issuing metric :

Lemma 26. The length of the maximal interval of integrability of the Ricci field -2 ric originating from any metric $g_{0}$ of positive scalar curvature is bounded from above by $2 /$ inf scal $\left(g_{0}\right)$.

Proof. Let $\rho$ be a positive real number ; the real function $f(t)=\frac{2 \rho}{2-\rho t}$ is a solution of the ordinary differential equation $\frac{\partial f}{\partial t}=\frac{f^{2}}{2}$ and $f(0)=\rho$, so that, according to the differential equation (3), the following differential inequality is satisfied

$$
\left(\frac{\partial}{\partial t}+D^{*} D\right)(\mathrm{scal}-f(t)) \geq \frac{1}{2}\left(\mathrm{scal}^{2}-f^{2}\right) .
$$

The maximum principle ([M3] chap. 3 - II) then implies that scal $(x, t) \geq$ $f(t)$, where $\rho$ is a lower bound for the scalar curvature of the initial metric $g_{0}$.

According to Proposition 21, the following differential inequality holds true

$$
\left(\frac{\partial}{\partial t}+D^{*} D\right)(Q-C(\eta) t) \leq 0
$$

Because of the parabolic maximum principle, this differential inequality implies that $\sup _{V}(Q-C(\eta), t)$ is non-increasing along any integral curve ; in particular, there is some constant, only depending on $\eta$ and the initial metric $g_{0}$ s.t.

$$
\sup _{M \times\left[0, T_{\max }\right)} Q \leq \sup _{M \times\{0\}} Q+C(\eta) T_{\max } \leq K\left(\eta, g_{0}\right) .
$$

Setting $\nu=(2 \eta)^{1 / 2}$, and using the Hölder inequality, this can be written

$$
\mid d \text { scal } \mid \leq \nu \mathrm{scal}^{3 / 2}+c\left(\nu, g_{0}\right),
$$

which concludes the proof of Corollary 25 .

\section{B. Geometric conclusion.}

According to Proposition 1, starting at any metric on any compact 4manifold there is a maximal integral curve $g_{t} \in[0, T)$ for the curvature 
field -2 ric such that $\lim _{t \rightarrow T}\|R\|_{\mathcal{C}^{0}}=+\infty$. Proposition 4 , the heart of the proof, asserts that the fundamental polynomial $P_{2}(R)$ evaluated at any $C-$, $C=\frac{1}{6},\left(C<\frac{1}{6}\right)$ weakly pinched curvature is non-positive (resp. negative). Because of the parabolic maximum principle this is enough to ensure that the maximum of the weak pinching is a non-increasing (resp. decreasing) function along the integral curve of the field -2 ric starting at any $1 / 6-$ (resp. $C-, C<1 / 6$ ) weakly pinched metric. In particular, the scialar curvature is an upper bound for the norm of the curvature all along such an integral curve and the maximum of the scalar curvature must blow up as $t$ approaches the upper bound $T$. Note that $T$ is finite if $g_{0}$ has positive scalar curvature, by Lemma 26.

Because of the control on the gradient of the scalar curvature that Corollary 25 provides, one proves that the infimum of the scalar curvature must also blow up at $T$.

Lemma 27. If the issuing metric is $C-\left(C<\frac{1}{6}\right)$ weakly pinched and has positive scalar curvature, then the scalar curvature becomes almost constant when approaching the singularity :

$$
\sup _{M \times\{t\}} \mid \text { scal }\left|/ \inf _{M \times\{t\}}\right| \text { scal } \mid \rightarrow 1 \text { as } t \rightarrow T .
$$

Proof. Because $g_{0}$ (and so $g_{t}$ ) is $C-\left(C<\frac{1}{6}\right)$ weakly pinched, Lemma 24 grants us a positive lower bound for the Ricci curvature : there exists $\varepsilon>0$, $\varepsilon$ only depending on $C$, such that for all $(t, x)$ in $[0, T) \times M$,

$$
\text { ric }\left(g_{t}\right)(x) \geq \varepsilon(n-1) \text { scal }\left(g_{t}\right)(x) g_{t}(x) \text {. }
$$

Then pick a positive real number $\nu$, small enough for $4 \pi^{2} \nu\left(1-\nu^{1 / 2}\right)^{-1}$ to be less than the previous $\varepsilon$. Clearly enough, since the (supremum of the) scalar curvature diverges at the singularity, there is some point $\tau$ in $[0, T)$ s.t. the constant $C\left(\nu, g_{0}\right)$ in (19) is bounded from above by $\nu \inf _{\theta \in[\tau, T)} \sup _{M \times\{\theta\}} \operatorname{scal}^{3 / 2}$. Let $p$ be a maximum of the scalar curvature on $\left(M, g_{\theta}\right)$; because of Corollary 25 , the scalar curvature is bounded from below by $\left(1-\nu^{1 / 2}\right) \sup _{V \times\{\theta\}}$ scal on the $g_{\theta}$-geodesic ball

$$
B_{p}=B\left(p, 2^{-1}\left(\nu \sup _{V \times\{\theta\}} \mathrm{scal}\right)^{-1 / 2}\right) .
$$


Inside the ball $B_{p}$, we have the following lower bound for the Ricci curvature

$$
\begin{aligned}
\text { ric } & \geq \varepsilon(n-1) \text { scal } g \geq \varepsilon(n-1)\left(1-\nu^{1 / 2}\right) \sup _{M \times\{\theta\}} \text { scal } g \\
& \geq 4 \pi^{2} \nu \sup _{M \times\{\theta\}} \text { scal } g,
\end{aligned}
$$

which, by invoking Myers theorem, implies that the conjugate distance to $p$ is at most $2^{-1}\left(\nu \sup _{M \times\{\theta\}} \text { scal }\right)^{-1 / 2}$. In particular, no geodesic starting at $p$ can be minimizing beyond $\partial B_{p}$, and the Hopf-Rinow theorem then says that $B_{p}$ covers the manifold $M$. As a consequence, we have proved

$$
\sup _{\left(M, g_{\theta}\right)} \text { scal } / \inf _{\left(M, g_{\theta}\right)} \text { scal } \leq\left(1-\nu^{1 / 2}\right)^{-1} \text { on }[\tau, T) .
$$

This establishes Lemma 27.

To conclude, let us remark that this is enough to prove that the weak pinching of any $C-(C<1 / 6)$ weakly pinched metric becomes arbitrarily small as $t \rightarrow T$. Indeed, since $C<1 / 6$, there is some real number $\beta$ in the interval $[1,2)$ such that $|\mathcal{D}|^{2} \mathrm{scal}^{-\beta}$ is non-increasing along the integral curve of the field -2 ric. This implies the bound

$$
|\mathcal{D}|^{2} \mathrm{scal}^{-2}=|\mathcal{D}|^{2} \mathrm{scal}^{-\beta} \mathrm{scal}^{-(2-\beta)} \leq\left(\sup _{M \times\{0\}}|\mathcal{D}|^{2} \mathrm{scal}^{-\beta}\right) \mathrm{scal}^{-(2-\beta)}
$$

which proves our claim, since $\inf _{M \times\{t\}}$ scal diverges at $T$ according to Lemma 27. Theorem 1 is then a corollary of any 4-dimensional differential sphere theorem since arbitrarily small weak pinching and almost constant scalar curvature obviously imply that the sectional curvatures are arbitrarily pinched (in the classical strong sense).

In fact one can prove a more precise statement without invoking any external result : after a proper rescaling (say such that $V\left(g_{t}\right)=V\left(g_{0}\right)$ ) the metrics $g_{t}$ converge exponentially towards a metric of constant curvature in all norms $\mathcal{C}^{(k)}, k \in \mathbb{N}$. See [M3, Chap. III - D p. 163-176].

\section{Rigidity : a classification of the limit cases.}

The object of this last part is to prove Theorem 2, i.e. to give a metric characterization of all 4-manifolds which are as pinched as $S^{1} \times\left(S^{3}\right.$, can $)$ or $\left(\mathbb{P}^{2}, \mathrm{~F}-\mathrm{S}\right)$ but neither diffeomorphic to $S^{4}$ nor $\mathbb{R} \mathbb{P}^{4}$. 
Proposition 28. The fundamental polynomial $P_{2}(R)$ (cf. (5)) is always non-positive at a 1/6-weakly pinched curvature and zero only if one of the following four cases holds :

1. $\|\mathcal{D}\|^{2}=0$, or

2. $\|\mathcal{D}\|^{2} \mathrm{scal}^{-2}=1 / 6,\left\|W^{+}\right\|=\left\|W^{-}\right\|=0$ and

$$
\operatorname{spec}(z)=\frac{\text { scal }}{4}\left(-1, \frac{1}{3}, \frac{1}{3}, \frac{1}{3}\right)
$$

$\left(S^{1} \times S^{3}\right.$-type curvature), or

3. $\|\mathcal{D}\|^{2} \mathrm{scal}^{-2}=\left\|W^{-}\right\|^{2} \mathrm{scal}^{-2}=1 / 6,\|Z\|=0$ and $\left\|W^{+}\right\|=: 0$ $\left(\overline{\mathbb{P}}^{2}\right.$-type curvature), or finally

4. $\|\mathcal{D}\|^{2}$ scal $^{-2}=\left\|W^{+}\right\|^{2}$ scal $^{-2}=1 / 6,\|Z\|=0$ and $\left\|W^{-}\right\|=0$ $\left(\mathbb{P}^{2}\right.$-type curvature).

Proof. This has essentially been observed in the course of the proof of Theorem 1, where we have noticed that the vanishing of some upper bound for the fundamental polynomial implies that one of the following holds: (1) the deviation $\mathcal{D}$ vanishes, or (2) the Ricci curvature is diagonal $(B=0)$ and $R$ is of type $\mathbb{P}^{2}$, or $\overline{\mathbb{P}}^{2}$, or (3) conformal curvature vanishes $(W=0)$ and $\widehat{Z}^{2}=\frac{\text { scal }^{2}}{36} i d_{\Lambda^{2}}$ (see Propositions 16, 17 and 18). To conclude we need only the following result.

Lemma 29. The only curvature tensors whose Weyl projection vanishes and whose Ricci part satisfies the identity $\widehat{Z}^{2}=\frac{\text { scal }^{2}}{36} i d_{\Lambda^{2}}$ are of $S^{1} \times S^{3}$ type, i.e. such that $W=0$ and $\mathrm{spec}$ (ric) $=\operatorname{scal}(0,1 / 3,1 / 3,1 / 3)$.

Proof. Being symmetric, the Ricci tensor is diagonalizable in a $g$-orthonormal frame $\left(e_{1}, \cdots, e_{4}\right)$; let $\left(z_{1}, \cdots, z_{4}\right)$ be the $z$-spectrum w.r.t. these eigenvectors. Then, the associated $\Lambda^{2}$-basis $e_{1} \wedge e_{2} \pm e_{3} \wedge e_{4}, e_{1} \wedge e_{3} \mp e_{2} \wedge$ $e_{4}, e_{1} \wedge e_{4} \pm e_{2} \wedge e_{3}$ is an eigenbasis for $\widehat{Z}^{2}$ with associated spectrum $\left(z_{1}+z_{2}-z_{3}-z_{4}\right)^{2},\left(z_{1}+z_{3}-z_{2}-z_{4}\right)^{2}$ and $\left(z_{1}+z_{4}-z_{2}-z_{3}\right)^{2}$.

The assumption in the lemma then implies that either $z_{1}+z_{2}-z_{3}-$ $z_{4}=z_{1}+z_{3}-z_{2}-z_{4}=z_{1}+z_{4}-z_{2}-z_{3}$ (and so $z_{1}=-\mathrm{scal} / 4$ and $\left.z_{2}=z_{3}=z_{4}=\mathrm{scal} / 12\right)$, or $z_{1}+z_{2}-z_{3}-z_{4}=z_{2}+z_{4}-z_{1}-z_{3}=$ $z_{1}+z_{4}-z_{2}-z_{3}$ (and so $z_{2}=-\mathrm{scal} / 4$ and $z_{1}=z_{4}=z_{2}=\mathrm{scal} / 12$ ), or 
A sharp characterizationof the 4-sphere in curvature terms

$z_{1}+z_{2}-z_{3}-z_{4}=z_{1}+z_{3}-z_{2}-z_{4}=z_{2}+z_{3}-z_{1}-z_{4}$ (and so $z_{4}=-\mathrm{scal} / 4$ and $\left.z_{1}=z_{2}=z_{3}=\mathrm{scal} / 12\right)$. This proves the lemma.

\section{A. The 1/6-weakly pinched metrics on 4-manifolds which are not diffeomorphic to $\left(S^{4}\right.$, can).}

Let us recall the following result in [M1] (see Theorem 1 there, and its proof).

Proposition 30. Positivity and non-negativity of the curvature operator are both preserved along any integral curve of the Ricci field -2 ric.

Any metric whose curvature is everywhere of type $S^{1} \times S^{3}$ (resp. of type $\mathbb{P}^{2}$ or $\overline{\mathbb{P}}^{2}$ ) is $1 / 6$-weakly pinched and has non-negative curvature operator of rank 3 (resp. 4). Since the base manifold is compact, and the rank is semi-continuous, there is, for any metric $g_{0}$ of type $S^{1} \times S^{3}$ (resp. $\mathbb{P}^{2}$ or $\overline{\mathbb{P}}^{2}$ ), a positive real $\varepsilon$ such that along the $[0, \varepsilon]$-segment of the integral curve of the field -2 ric originating from $g_{0}$, the curvature is non-negative and of rank at least 3 (resp. 4).

Lemma 31. Any 1/6-weakly pinched 4-manifold of positive scalar curvature whose weak pinching at some point is smaller than $1 / 6$, is diffeomorphic either to the standard $S^{4}$ or its standard $\mathbb{Z}_{2}$-quotient $\mathbb{R P}^{4}$.

Proof. By the proof of Theorem 1, weak pinching $|\mathcal{D}|^{2} \mathrm{scal}^{-2}$ satisfies the following differential inequality

$$
\left(\frac{\partial}{\partial t}+D^{*} D\right)|\mathcal{D}|^{2} \mathrm{scal}^{-2}+\left(d\left(|\mathcal{D}|^{2} \mathrm{scal}^{-2}\right), \omega\right) \leq 0,
$$

for some 1-form $\omega$ on $M$, provided that the initial metric is $1 / 6$ weakly pinched. Let us consider a $1 / 6$-weakly pinched metric. If its weak pinching at some point is less than $1 / 6$, then the (global) weak pinching is less than $1 / 6$ on some integral arc $(0, \varepsilon]$ originating from $g_{0}$. This follows from the strong maximum principle for parabolic type equations (cf. [M3 ; chap. 3-II B]). To conclude, one can invoke Theorem 1 for any metric $g_{t}, t \in(0, \varepsilon]$, to prove that the underlying manifold is in fact diffeomorphic to either the standard $S^{4}$ or its only standard quotient $\mathbb{R} \mathbb{P}^{4}$.

Corollary 32. Any compact 4-manifold which admits a 1/6- weakly pinched metric of positive scalar curvature whose curvature at some point is 
neither of type $S^{1} \times S^{3}$ nor of type $\mathbb{P}^{2}\left(\overline{\mathbb{P}}^{2}\right)$ must be diffeomorphic to either the standard $S^{4}$ or the standard $\mathbb{R} \mathbb{P}^{4}$.

Proof. If at some point the weak pinching is less than $1 / 6$, the corollary follows immediately from the previous lemma. If not, the weak pinching is constant and the differential equation satisfied by the weak pinching can be written, according to Theorem 1 and Proposition 28,

$$
\frac{\partial}{\partial t}|\mathcal{D}|^{2} \operatorname{scal}_{\mid t=0}^{-2}<0
$$

in a neighbourhood of a point $p$ where the curvature is neither of type $S^{1} \times S^{3}$ nor $\mathbb{P}^{2}\left(\overline{\mathbb{P}}^{2}\right)$; in particular for some positive real number $\varepsilon$ the weak pinching at $p$ and $t=\varepsilon,|\mathcal{D}|^{2}$ scal $^{-2}(\varepsilon, p)$, is less than $1 / 6$ when the global pinching $\sup |\mathcal{D}|^{2}$ scal $^{-2}$ is at most $1 / 6$, according to Theorem 1 . Lemma 31 then $\varepsilon \times M$ concludes the proof.

Note that a trivial continuity argument proves that any metric (on a connected manifold) whose curvature at any point is of one of the three types $S^{1} \times S^{3}, \mathbb{P}^{2}$ or $\overline{\mathbb{P}}^{2}$, is either everywhere of type $S^{1} \times S^{3}$, everywhere of type $\mathbb{P}^{2}$, or everywhere of type $\overline{\mathbb{P}}^{2}$.

Corollary 33. Let $g_{0}$ be a metric on a smooth compact 4-manifold whose curvature is of type $\mathbb{P}^{2}$ or $\left(\overline{\mathbb{P}}^{2}\right)$. Let us assume that the rank at sorne point on the manifold of the curvature of some metric $g_{t}$ on the integral curve of the Ricci field, -2 ric, issuing from $g_{0}$, is larger than 4. Then the manifold must be diffeomorphic to either the standard $S^{4}$ or the standard $\mathbb{R P}^{4}$.

Proof. The metric $g_{t}$ for which there is some point where the curvature rank is larger than 4 can neither be of type $\mathbb{P}^{2}$ or $\overline{\mathbb{P}}^{2}$ (rank 4 ) nor of type $S^{1} \times S^{3}$ (rank 3 ) at that point. Corollary 33 therefore follows from Corollary 32.

Corollary 34. If a compact 4-manifold is not diffeomorphic to the standard $S^{4}$ nor to its standard quotient $\mathbb{R P}^{4}$ and admits some metric $g_{0}$ whose curvature is everywhere of type $\mathbb{P}^{2}$ or of type $\overline{\mathbb{P}}^{2}$, then there is some positive real number $\varepsilon$ such that the rank of the curvature of any metric along the integral segment $[0, \varepsilon]$ of the Ricci field -2 ric issuing from $g_{0}$, is constant (and equal to 4).

Proof. Because of the semi-continuity property for the rank, this is just a reformulation of the previous statement. 
Lemma 35. Let $M$ be a compact 4-manifold which is not diffeomorphic to the standard $S^{4}$ nor to its standard $\mathbb{Z}_{2}$-quotient. If $M$ can be equipped with some metric $g_{0}$ whose curvature is everywhere of type $S^{1} \times S^{3}$, then there is a positive real number $\varepsilon$ such that the rank of the curvature of every metric along the integral segment $[0, \varepsilon]$ of the Ricci field, -2 ric, originating from $g_{0}$, is constant and equal to 3 .

Proof. By continuity, there is some integral segment $[0, \varepsilon], \varepsilon>0$, of the Ricci field such that the metric $g_{t}$ has curvature whose rank is at least 3 and whose type is neither $\mathbb{P}^{2}$ nor $\overline{\mathbb{P}}^{2}$, for all $\mathrm{t}$ in the interval $[0, \varepsilon]$. If at some point $(t, P)$ in $[0, \varepsilon] \times M$ the rank of the curvature is more than 3 , then the curvature at this point is neither of type $S^{1} \times S^{3}, \mathbb{P}^{2}$, nor $\overline{\mathbb{P}}^{2}$. We can conclude, according to Corollary 32 , that the manifold is a standard (quotient of) $S^{4}$.

Lemma 36. If the rank of the curvature operators of all metrics in an integral segment $[0, \varepsilon]$ of the Ricci field, -2 ric, originating from a metric with a non-negative curvature operator is constant (as a function on $[0, \varepsilon] \times M$ ), then the kernels of the curvature operators form a parallel distribution in $\Lambda^{2} T^{*} M$.

Proof. (Compare with Lemma 8.2 and Theorem 8.3 in [H2].) Let us consider a smooth section $\omega_{t}$ in ker $\widehat{R}\left(g_{t}\right)$. From the equation

$$
\widehat{R}_{g_{t}}\left(\omega_{t}, \omega_{t}\right)=0,
$$

we derive the following two identities :

$$
\begin{aligned}
\left(\frac{\partial \widehat{R}}{\partial t}\right)\left(\omega_{t}, \omega_{t}\right)= & -2 \widehat{R}\left(\omega_{t}, \frac{\partial \omega_{t}}{\partial t}\right)=0 ; \\
\left(\left(D^{*} D \widehat{R}\left(g_{t}\right)\right)\left(\omega_{t}\right), \omega_{t}\right)= & 4\left(\left(D \widehat{R}\left(g_{t}\right)\right)\left(\omega_{t}\right), D \omega_{t}\right) \\
& +2\left(\widehat{R}\left(g_{t}\right)\left(D \omega_{t}\right), D \omega_{t}\right) \\
= & -2\left(\widehat{R}\left(g_{t}\right)\left(D \omega_{t}\right), D \omega_{t}\right) .
\end{aligned}
$$

According to the above two identities, equation (1) for the curvature along an integral curve of the Ricci flow, which we recall in the condensed form

$$
\frac{\partial R}{\partial t}+D^{*} D R=p(R, g)
$$


becomes

$$
p(R, g)\left(\omega_{t}, \omega_{t}\right)=-2 \widehat{R}_{g_{t}}\left(D \omega_{t}, D \omega_{t}\right) .
$$

In [M1], we proved that $p\left(\widehat{R}, g_{t}\right)$ is non-negative on the kernel of $\widehat{R}_{g_{t}}$ provided $g_{t}$ has a non-negative curvature operator. According to Proposition $30, g_{t}$ has a non-negative curvature operator when the originating metric $g_{0}$ does. Identity (20) then shows that $D \omega_{t}$ lies in the kernel of the curvature.

\section{B. Metrics with $S^{1} \times S^{3}$-type curvature.}

Proposition 37. Any compact Riemannian 4-manifold which is not diffeomorphic to (the standard) $S^{4}$ nor to its standard quotient $\mathbb{R P}^{4}$ and whose curvature is everywhere of type $S^{1} \times S^{3}$ is (isometric to) a quotient of $\mathbb{R} \times\left(S^{3}, \lambda\right.$ can $), \lambda \in \mathbb{R}_{+}^{*}$.

Proof. Assume $M$ is neither diffeomorphic to $S^{4}$ nor to the standard $\mathbb{R P}^{4}$. The rank of the curvature operator on $M$ must then be constant and equal to 3 on some interval $[0, \varepsilon], \varepsilon>0$, according to Lemma 35 . This implies that the curvature is parallel :

Let $X_{1}$ be a unit vector field on $\left(M, g_{t}\right)$ in the kernel of the Ricci curvature (a 1-dimensional distribution in $T M$ ) and let $X_{2}$ and $X_{3}$ be two unit vector fields, orthogonal to one another and both orthogonal to $X_{1}$. Then. $X_{1} \wedge X_{2}$ and $X_{1} \wedge X_{3}$ are in the kernel of the curvature operator $\widehat{R}_{g_{t}}$, the $\Lambda^{2} T M$ subspace generated by $\left(X_{1} \wedge X_{i}\right)_{i=2}^{4}$, where $\left(X_{i}\right)_{i=1}^{4}$ is an orthonormal frame, and $X_{2} \wedge X_{3}$ is orthogonal to the kernel. The second Bianchi identity and our assumption that the curvature is of type $S^{1} \times S^{3}$ imply $\widehat{R}=f \Pi_{(\mathrm{ker} R)^{\perp}}$, where $f$ is a smooth function on the manifold s.t. $d f\left(X_{1}\right)\left(X_{2} \wedge X_{3}, Y \wedge Z\right)=0$ for all vector fields $Y$ and $Z$ on $M$ : in particular, grad $f$ is orthogonal to ker ric in $T M$.

The same argument with three orthogonal unit vector fields, all of which being orthogonal to $X_{1}$, shows that $0 \equiv d f(\cdot) \wedge \cdot \wedge{ }_{i\left(\operatorname{ker} R_{t}\right)^{\perp}}$, i.e. that grad $f$ also lies in the kernel of the Ricci trace. Thus $f$ must be a constant - as claimed.

Being parallel, the line-bundle ker ric is trivial on the universal covering $\widetilde{M}$, and admits some global parallel unit section whose integral curves are geodesics ; these geodesics are without conjugate pairs since the sectional 
curvature vanishes on any 2-plane tangent to the geodesic - according to the definition of $S^{1} \times S^{3}$-type curvature. Since the universal cover, $\widetilde{M}$, is a symmetric space, all previous geodesics are rays. According to J. Cheeger and D. Gromoll's classification of complete, non-negatively curved manifolds this implies that $\widetilde{M}$ is isometric to some product $\mathbb{R}^{k} \times N$, where $N$ is compact, without any rays, and $k$ is at least 1 . The definition of curvature of type $S^{1} \times S^{3}$ then implies that $k$ is in fact one and that $N$ is a sphere of constant (sectional) curvature.

\section{Metrics with $\mathbb{P}^{2}\left(\overline{\mathbb{P}}^{2}\right)$-type curvature.}

Proposition 38. Any compact Riemannian 4-manifold whose curvature is everywhere of type $\mathbb{P}^{2}\left(\overline{\mathbb{P}}^{2}\right)$ is isometric to $\mathbb{P}^{2}$ (resp. $\left.\overline{\mathbb{P}}^{2}\right)$, up to a scalar factor, or diffeomorphic to either $S^{4}$ or its standard quotient $\mathbb{R P}^{4}$.

Note. Since any metric whose curvature is everywhere of type $\left(\mathbb{P}^{2}, \mathrm{~F}-\mathrm{S}\right)$ or $\left(\overline{\mathbb{P}}^{2}, \mathrm{~F}-\mathrm{S}\right)$ is Einstein, the second Bianchi identity shows that its curvature tensor is, up to a global scalar multiple, the curvature of the Fubini-Study metric. Proposition 38 can then be deduced from a a theorem by F.Tricerri and L.Vanhecke, which states that any metric whose curvature at any point is the curvature of an irreducible symmetric space, is locally isometric to the model space. The reason why we here give an alternate proof is that it also applies to curvatures of type $S^{1} \times S^{3}$ (see Proposition 37), whereas Tricerri and Vanhecke's approach does not apply to reducible symmetric spaces with a non-trivial Euclidean factor. In fact, their statement is even false for $\mathbb{H}^{2} \times \mathbb{R}$.

Proof. Assuming that the manifold $M$ is neither diffeomorphic to $S^{4}$ nor to $\mathbb{R P}^{4}$, let us introduce the orientation covering, $\stackrel{\vee}{M}$, of $M$; according to Corollary 34 , the rank of the curvature is constant and equal to 4 on an integral arc $[0, \varepsilon], \varepsilon>0$, and Lemma 36 asserts that the distribution of the kernel of the curvature operator is parallel. Since the Hodge decomposition is parallel and the kernel of the curvature of the initial metric $g_{0}$ lies either in $\Lambda^{+}$(or in $\Lambda^{-}$), according to the choice of orientation on $\stackrel{\vee}{M}$, the distribution ker $\widehat{R}\left(g_{t}\right)$ is parallel in $\Lambda^{+}$(resp. $\Lambda^{-}$).

Equivalently (ker $\left.\widehat{R}\left(g_{t}\right)\right)^{\perp}$ defines a parallel line distribution, $L$, in $\Lambda^{+}$ (resp. $\Lambda^{-}$). Since the curvature is everywhere of type $\mathbb{P}^{2}\left(\overline{\mathbb{P}}^{2}\right)$, all metrics $g_{t}$ are Einstein and all sectional curvatures are positive . Being compact, even- 
dimensional, oriented and of positive curvature, $\stackrel{\vee}{M}$ is simply connected and the line bundle $L$ is trivial. In particular there is a global never-vanishing parallel section, $\sigma$, in $L$.

Lemma 39. The parallel trivializing section $\sigma$ is symplectic.

Proof. Since $L$ is a subbundle of $\Lambda^{+}\left(\right.$resp. $\left.\Lambda^{-}\right)$,

$$
\sigma \wedge \sigma=\sigma \wedge * \sigma=|\sigma|^{2} d \text { Vol (resp. }=-\sigma \wedge * \sigma=-|\sigma|^{2} d \text { Vol) }
$$

This proves that $\sigma$ is of maximal rank.

To go from the symplectic 2-form $\sigma$ to the Kähler structure we view $\sigma$ as a field of (skew-) Hermitian endomorphims which we can diagonalize (over $\mathbb{C})$; the almost complex structure associated to the $\frac{\pi}{2}$-rotation in each $(\mathbb{C}-)$ eigenline is then parallel, and hence integrable. In our 4-dimensional setting this reduces to the observation that any element in $\Lambda^{+}\left(T_{p} \stackrel{\vee}{M}\right), p$ a point in $M$, admits a decomposition of the following form: $u \wedge v+*(u \wedge v)$, for orthogonal vectors $u$ and $v$ in $T_{p} \stackrel{\vee}{M}$. Since $*$ is an isometry, this can be written $\lambda(u \wedge v+w \wedge t), \lambda \in \mathbb{R}_{+}^{*}$, where $(u, v, w, t)$ is an orthogonal basis for the tangent plane $\left(T_{p} M, g_{t}(p)\right)$ under consideration. We then define $J$ by $J u=v, J v=-u, J w=t$ and $J t=-w$, a definition which is clearly independant of the choice of the splitting $\lambda(u \wedge v+w \wedge t)$. Moreover $J^{2}$ is equal to $-\mathrm{id}$ and is an isometry by construction. As a consequence, $J$ is skew-symmetric. To extend the almost complex structure $J$ to all of $\stackrel{v}{M}$ we transport the vectors $u, v, w$ and $t$ parallel-wise along any curve. Though the resulting fields $U, V, W$ and $T$ may well depend on the path, $\lambda(U \wedge V+W \wedge T)=\sigma$ is nevertheless well defined : set, as before, $J U=$ $V, \cdots$, to get the expected complex structure.

Recalling the elementary result (see [Ber] Prop. 7.5) that any compact Kähler - Einstein manifold with positive sectional curvature is, up to a scalar multiple, isometric to the projective space of the same dimension, equipped with the Fubini - Study metric, we conclude that $\left.\stackrel{v}{M}=\underset{v}{\mathbb{P}^{2}}, \mathrm{~F}-\mathrm{S}\right)$ (resp. $\overline{\mathbb{P}}^{2}$, F-S). Since $\mathbb{P}^{2}$ admits no smooth proper quotient, $M=M=\left(\mathbb{P}^{2} ; \mathrm{F}-\mathrm{S}\right)$.

\section{References.}


[Ber] M. Berger, Sur les variétés d'Einstein compactes, Compte-Rendus de la $\mathrm{III}{ }^{e}$ réunion du groupement des mathématiciens d'expression latine CBRM (1966).

[CG1] J. Cheeger and D. Gromoll, The splitting theorem for manifolds of non negative Ricci curvature, J. Diff. Geometry, 6 (1).

[CG2] J. Cheeger and D. Gromoll, On the structure of complete manifolds of non negative curvature, Ann. Math. 96 (3), 413-443.

[C-S1] S.E. Cappel and J.L. Shaneson, On 4-dimensional surgery and applications, Com. Math. Helv. 46 (1971), 500-528.

[C-S2] S.E. Cappel and J.L. Shaneson, Some new four manifolds, Ann. of Math. 104 (1976), 61-72.

[F] M.H. Freedman, The topology of four-dimensional manifolds, J. Diff. Geometry, 17 (1982), 357-453.

[H1] R. Hamilton, 3-manifold with positive Ricci curvature, J. Diff. Geometry, 17 (1982), 255-306.

[H2] R. Hamilton, 4-manifold with positive curvature operator, J. Diff. Geometry, 24 (1986), 153-179.

[L] J. Lannes, Un faux espace projectif réel de dimension 4, Séminaire Bourbaki $\mathrm{n}^{\circ} 527(78 / 79)$.

[M1] C. Margerin, Pointwise pinched manifolds are space forms, in Proceedings of Symposia in Pure Mathematics, 44 (1986) [Arcata : Geometric Measure Theory July 1984].

[M2] C. Margerin, Un théorème optimal pour le pincement faible en dimension 4 ("A sharp theorem for weakly-pinched 4-manifolds"), C.R. Acad. Sc. Paris t. 303 série I n ${ }^{\circ} 17$ (1986) and Preprint Ecole Polytechnique (1986).

[M3] C. Margerin, Déformations globales de structures riemanniennes I et II, Preprints Ecole Polytechnique n ${ }^{\circ}$ 954, 1004, 1006, 1073 and 1100.

RECEIVEd April 12, 1996 AND REVISEd July 30, 1996.

Ecole Polytechnique

F - 91128 Palaiseau CEDEX 